تأثير تغطية التربة والتركيز وعدد مرات الرش بالكبريت السائل ZOLFAST في المكونات

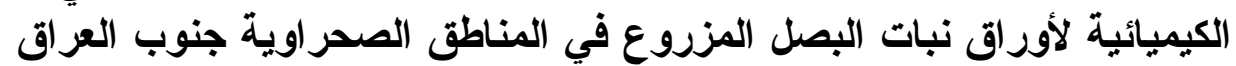

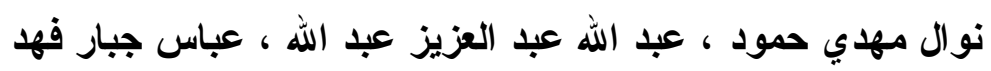

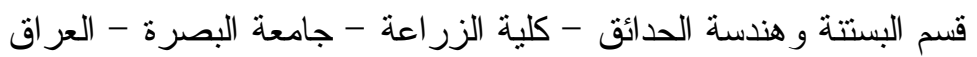

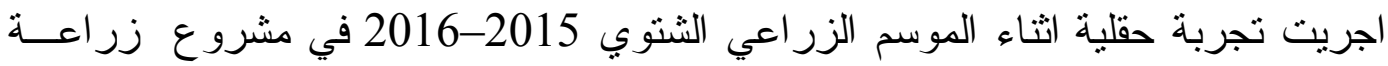

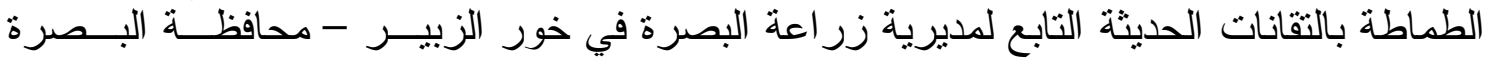

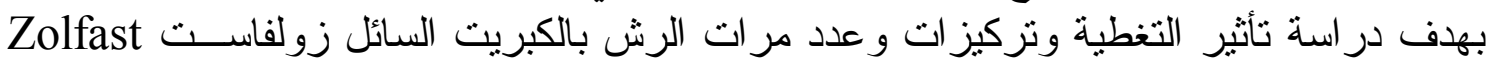

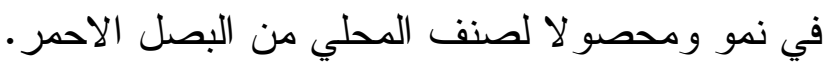

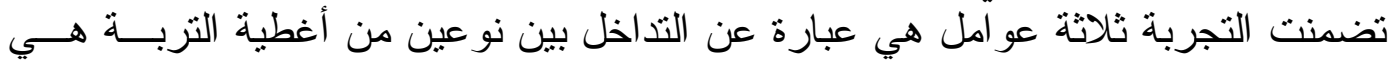

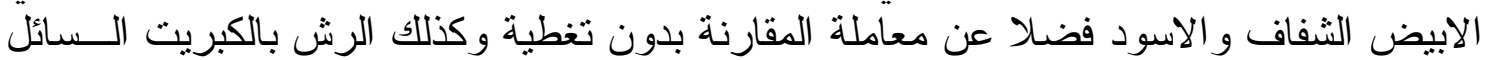

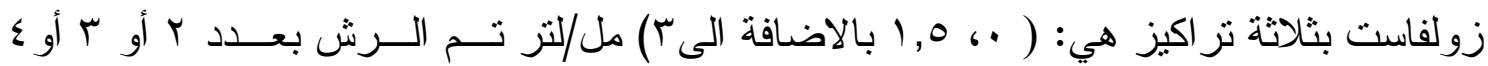

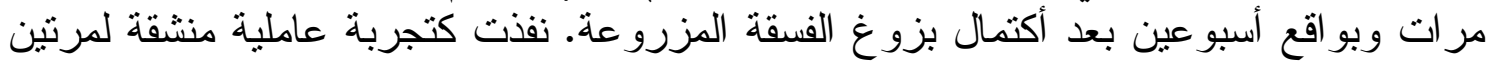

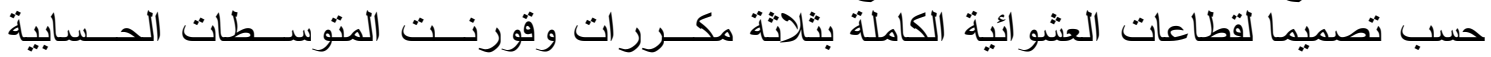

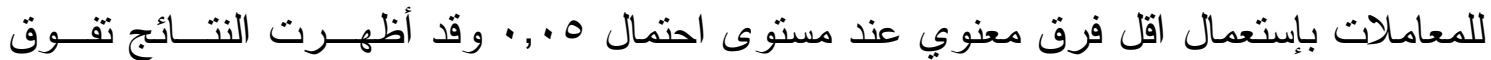

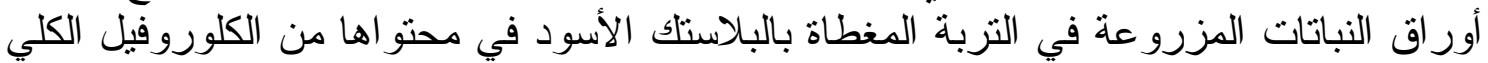

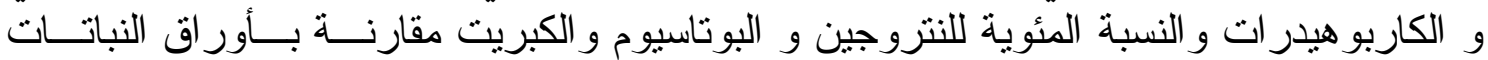

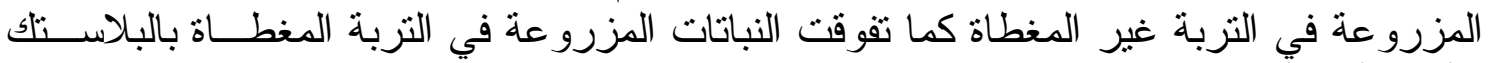

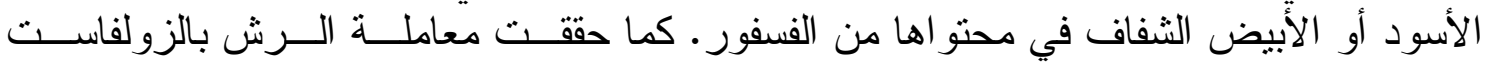

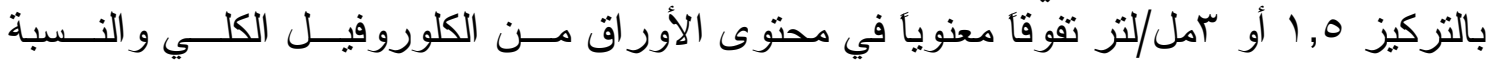

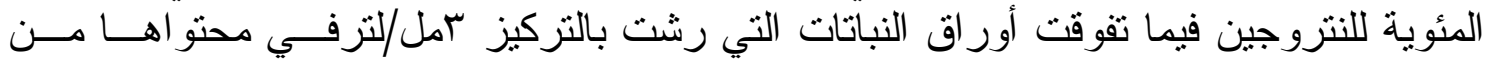

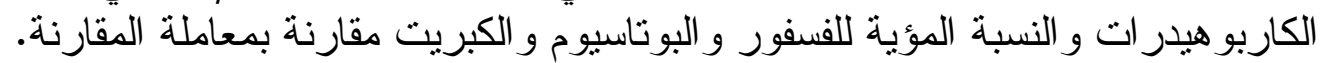

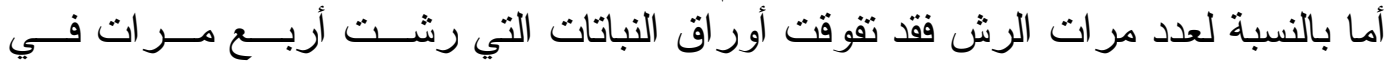

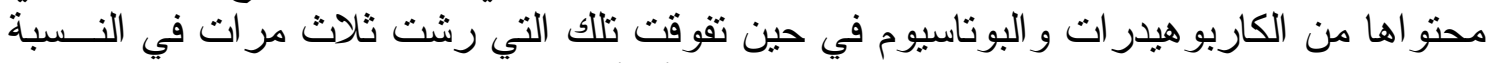

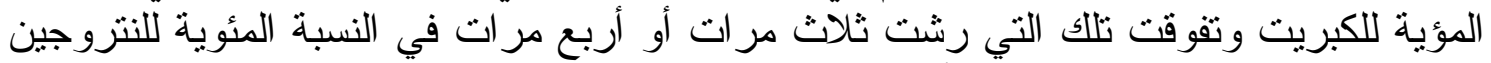

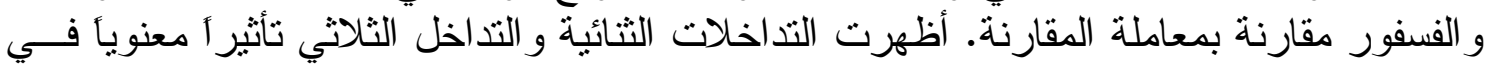
معظم الصفات قيد الدر اسة. المقدمة

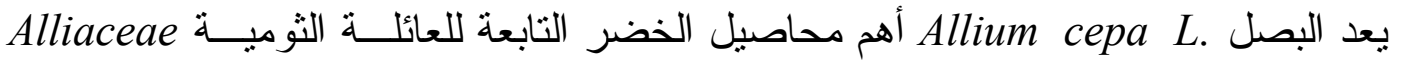

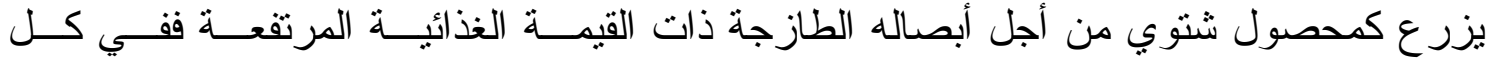

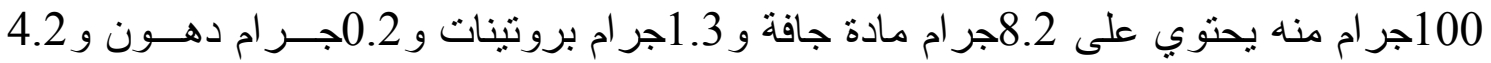

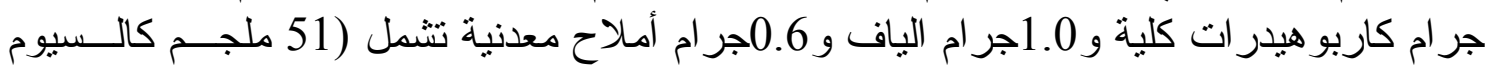

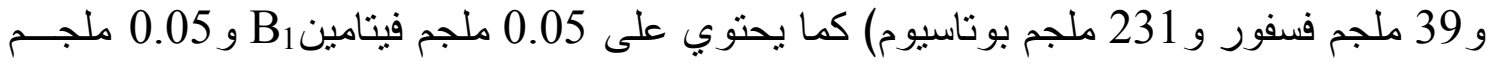

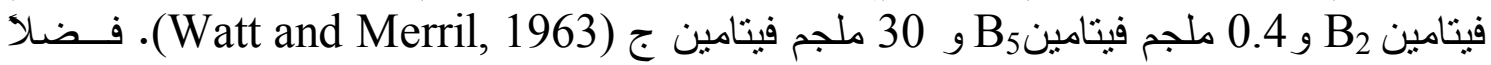

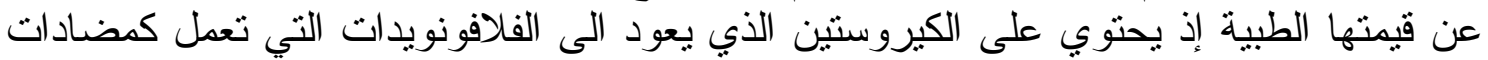

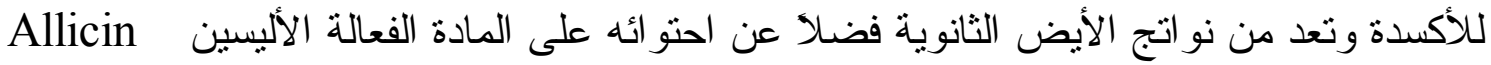

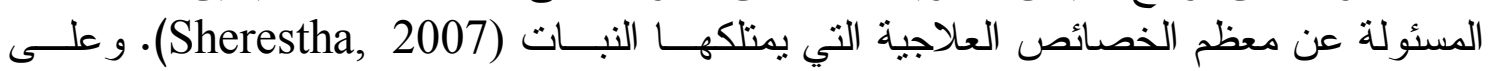
الرغم من أهمية المحصول الأقتصادية و الغذائية و الطبية إلا أن المساحة المزرو عة بالبــصل لا لا 


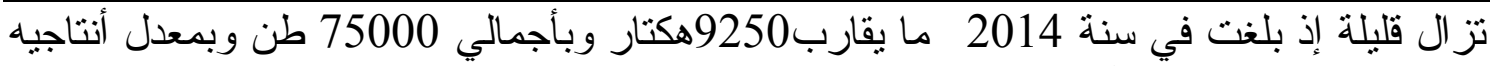

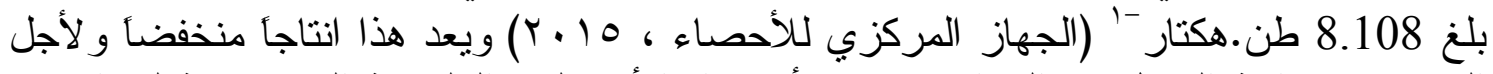

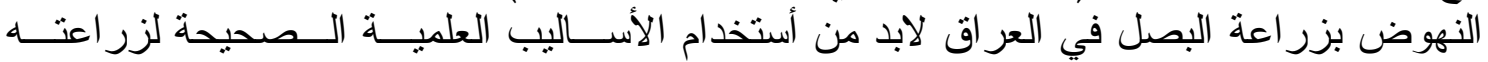

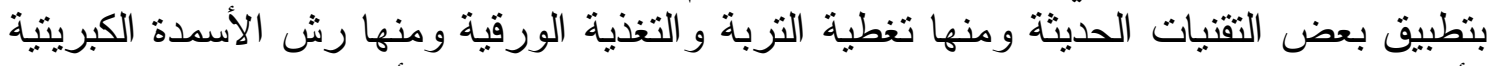

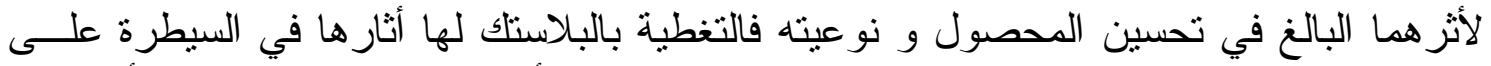

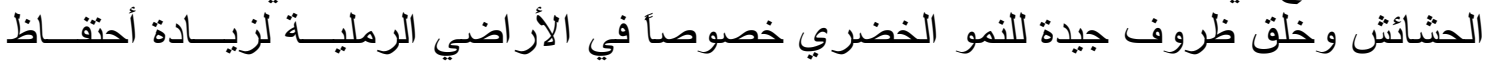

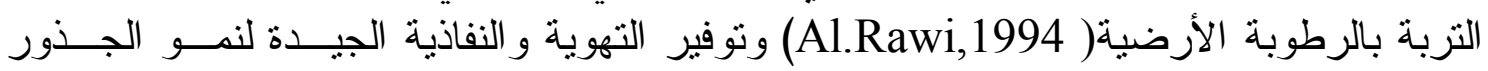

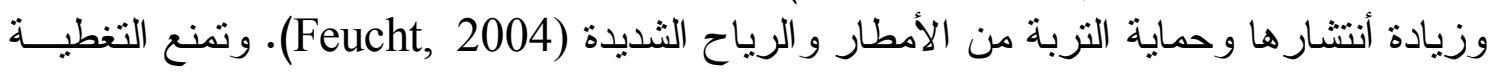

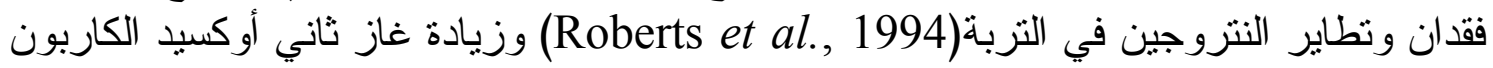

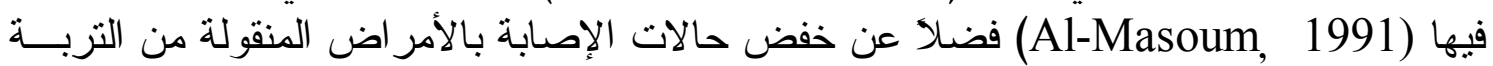
(Ctreck et al., 1996)

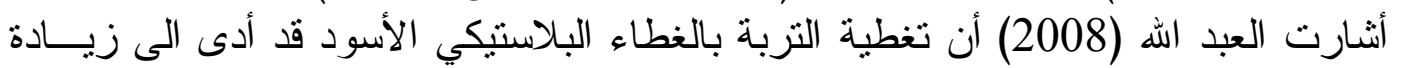

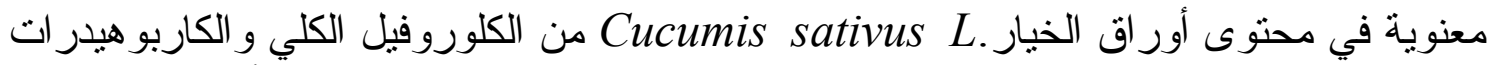

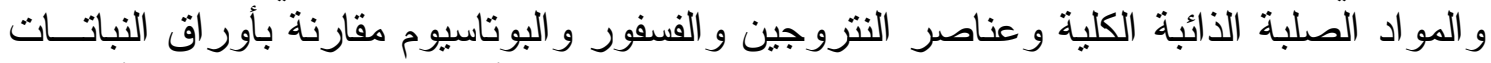

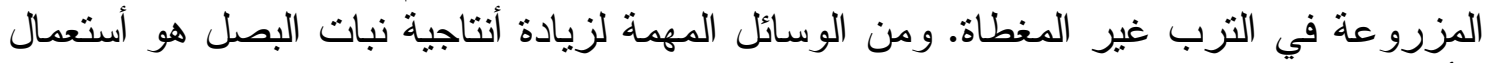

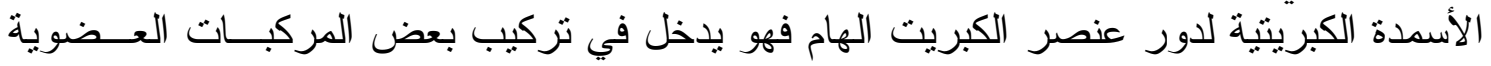

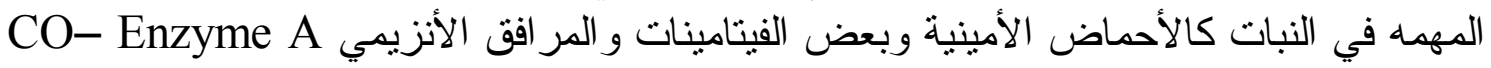

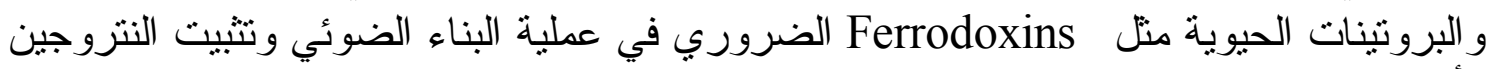

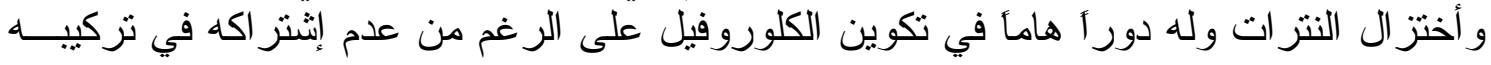

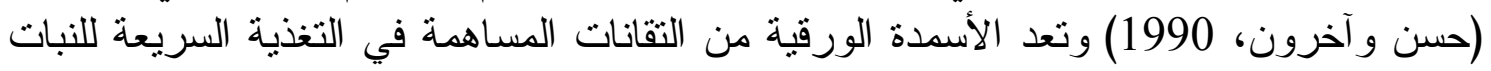

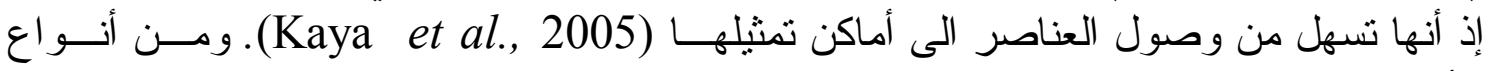

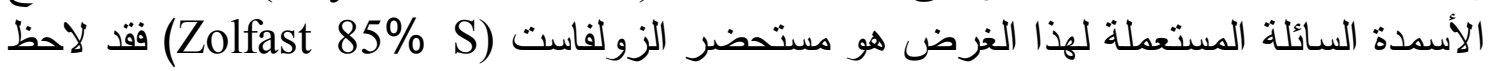

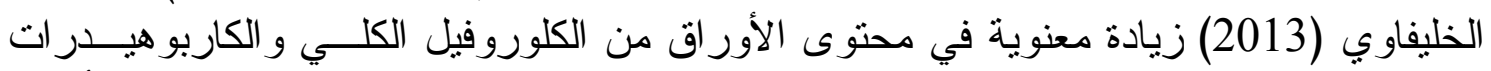

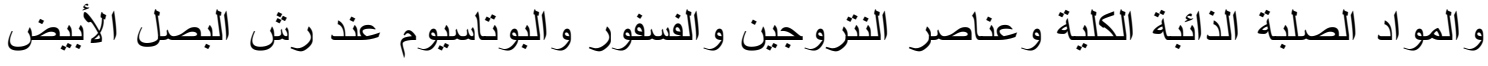

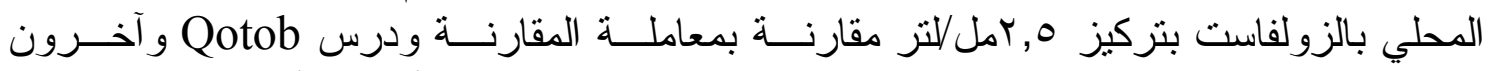

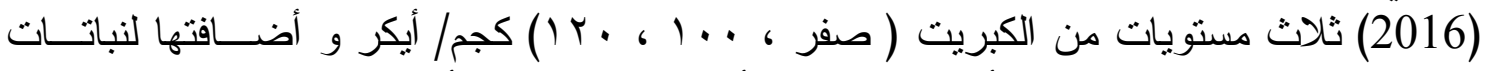

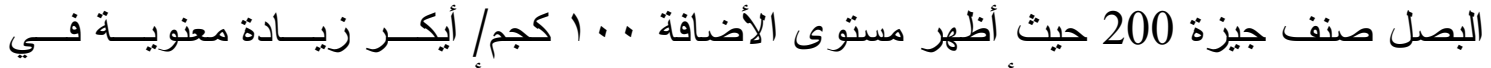

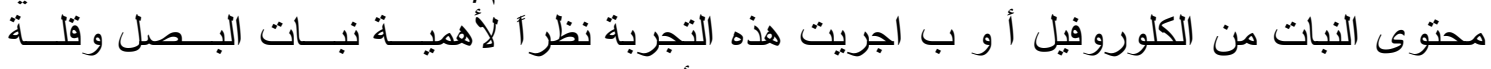

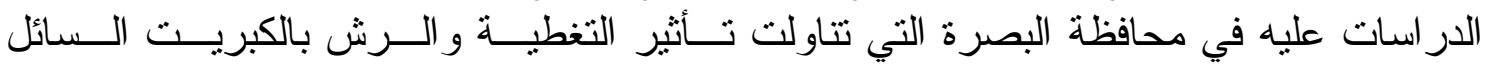

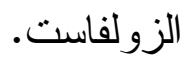

\section{المواد وطر ائق العمل}

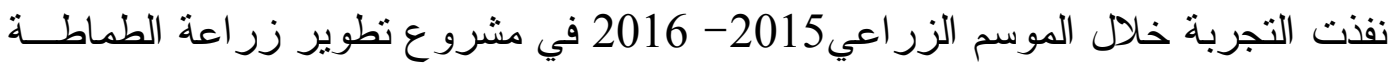

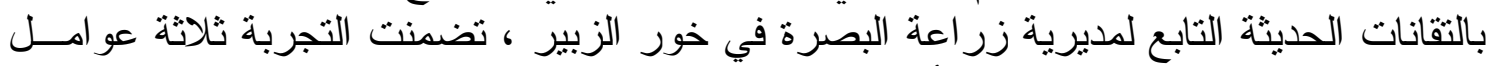

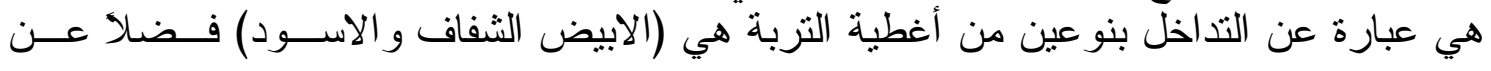

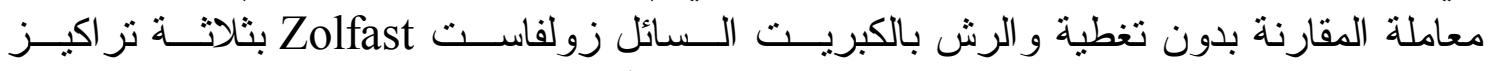

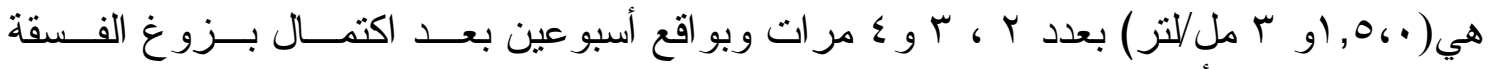

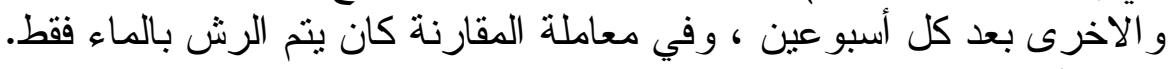

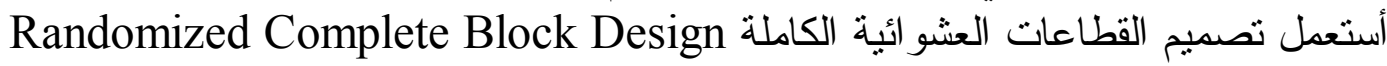

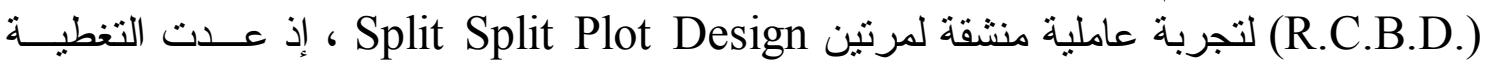




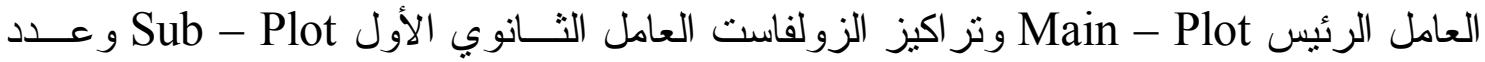

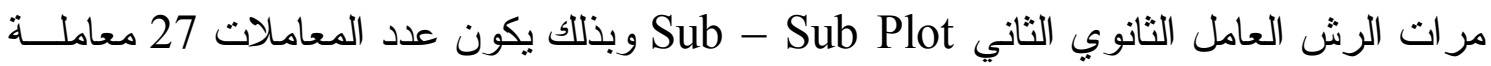

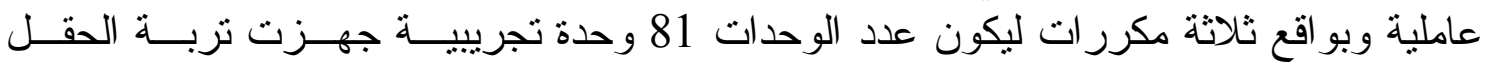

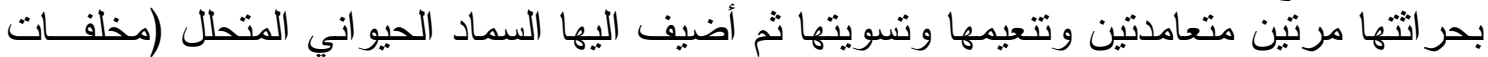

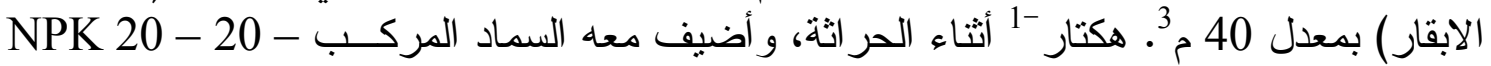

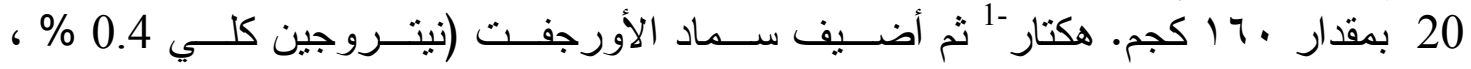
نيتزوجين عضوي 3.6 \% ، فوسفات 3 \% ، ، بوتاسيوم 2.5 \% ، مغنبسيوم 1 \% \% بالاضافة الى

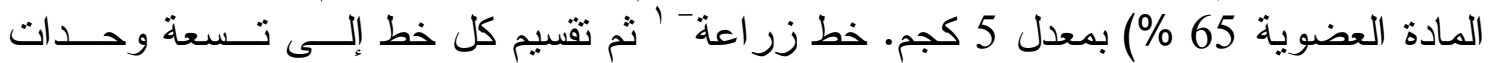

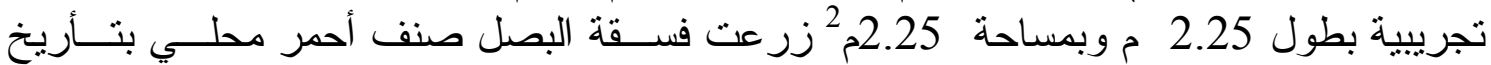

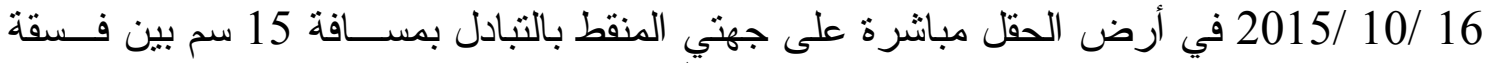

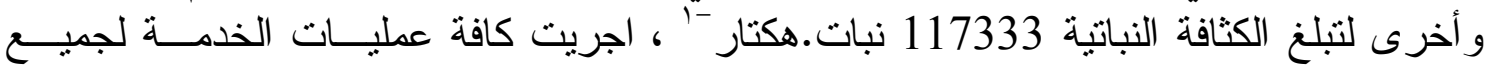

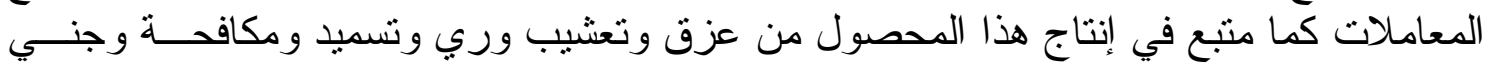

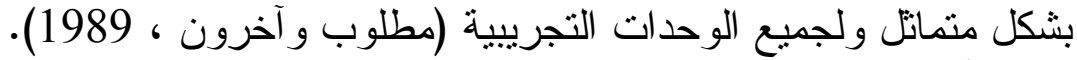

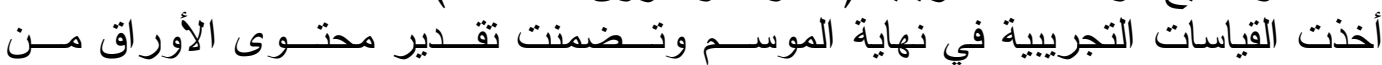

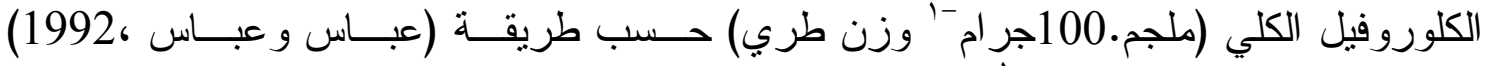

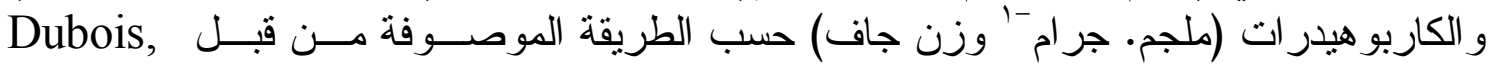

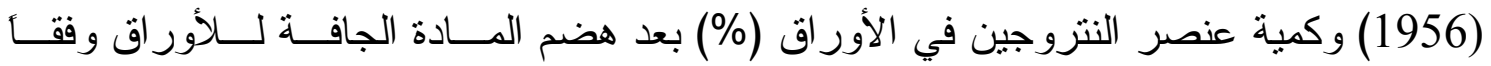
لطريقة (Cresser and Parsons 1979) قدر النتروجين الكلي في العينات الورقية المهضومة

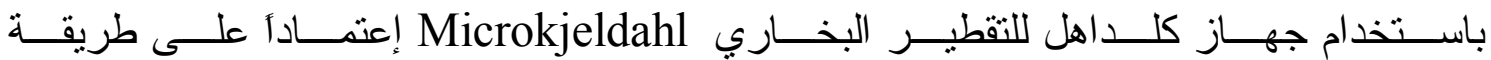
.(Page et al., 1982)

وبالنسبة للفسفور(\%) نم التقدير في العينات الورقية المهضومة باستخدام جهاز المطيــاف

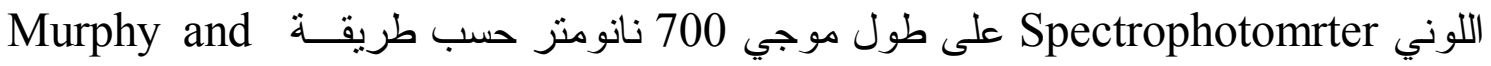

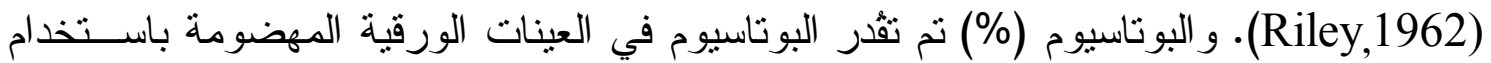

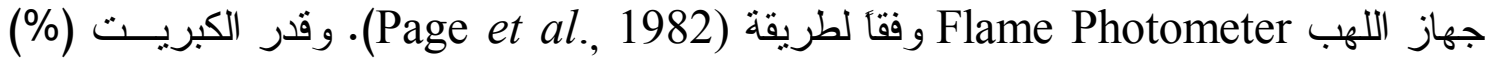
بالطريقة اللونية بعد تثكيل العكارة (\%) حسب طريقـة (1966، Chaudhary Cornfield

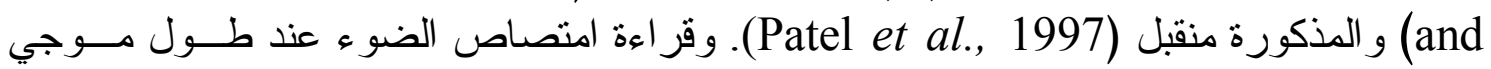

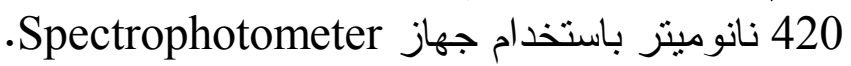

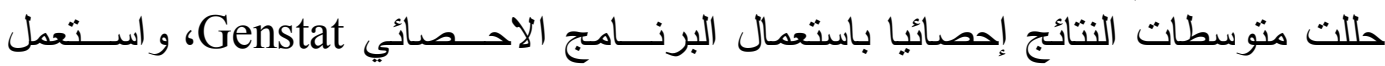

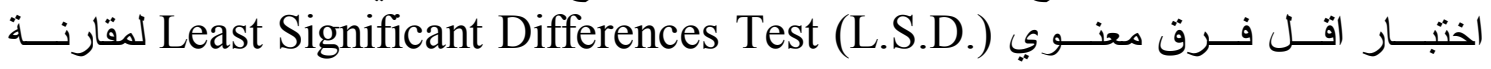
المتوسطات عند مستوى احتمال 0.05 (الر اوي وخلف الله، .919.D). النتائج و المناقثة

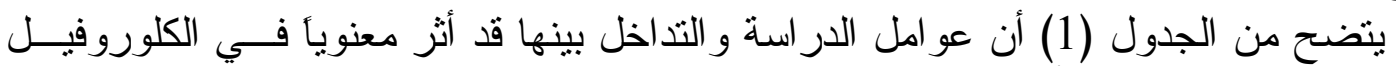

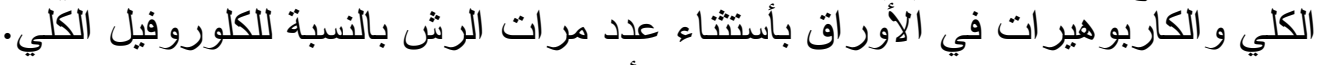

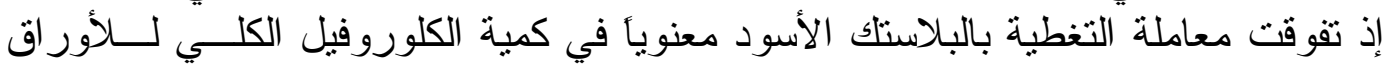

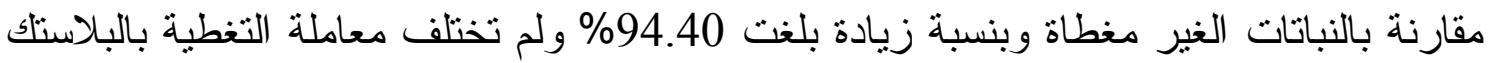

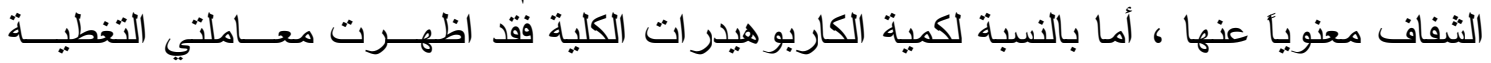

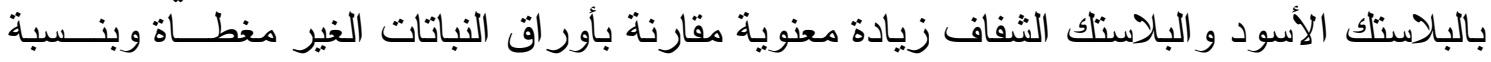

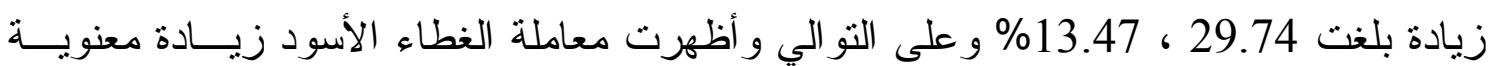

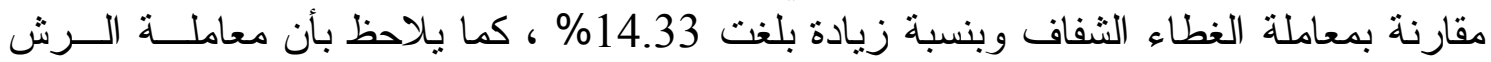




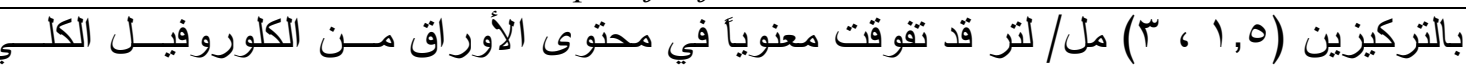

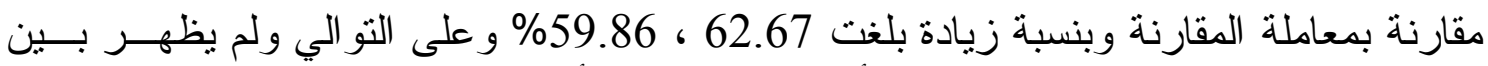

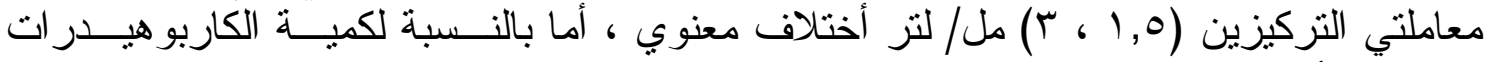

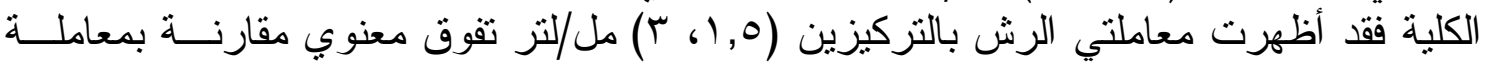

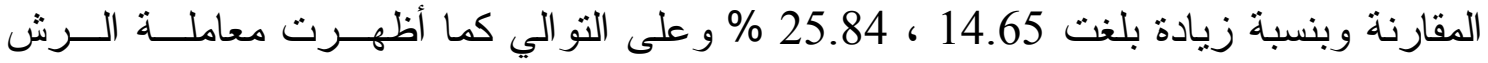

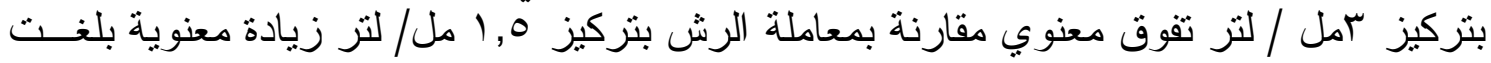

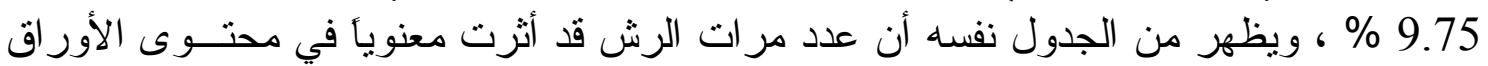

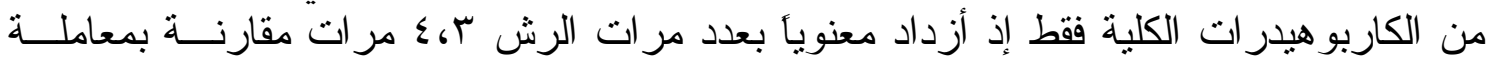

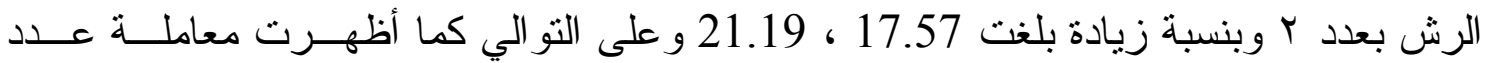

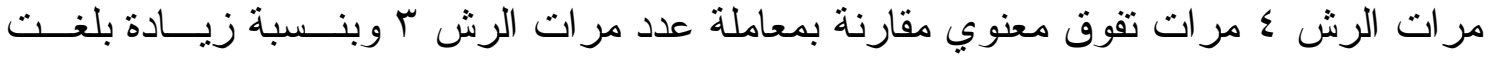

أما التداخلات فقد أظهرت تأثثر آ معنوياً لهاتين الصفتين إذ تفوق تداخل التغطية بالبلاســاتك

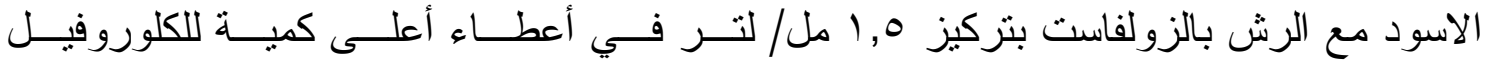

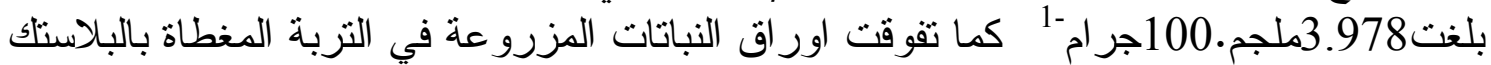

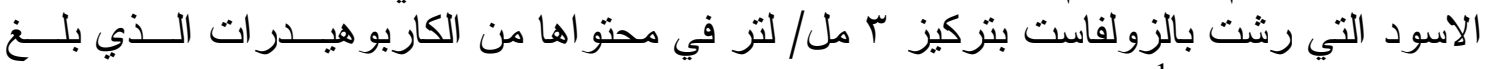
15.28 ملجم. جر ام-1-

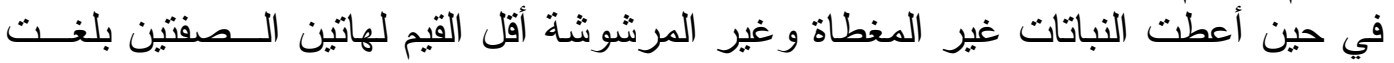

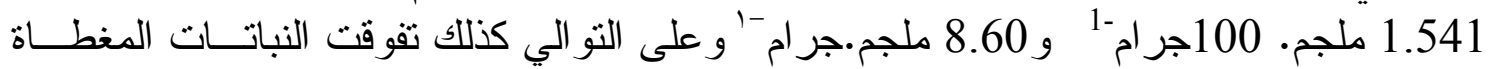

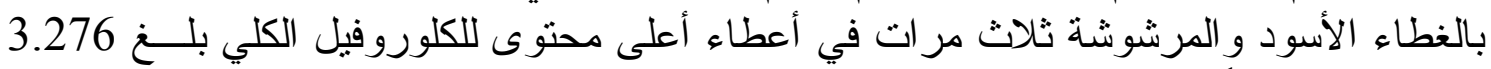

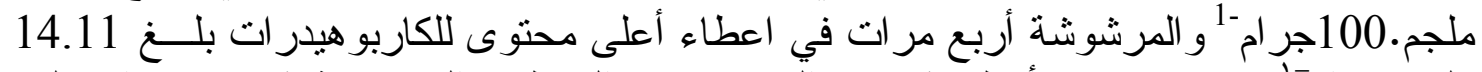

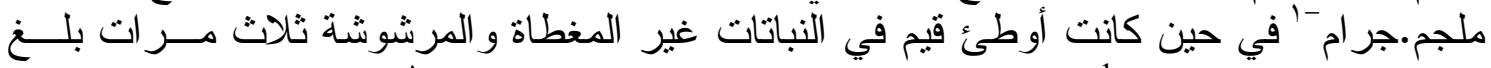

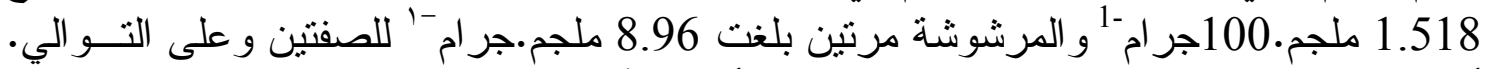

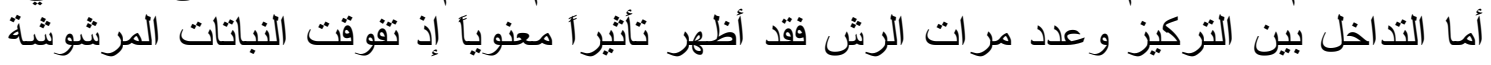

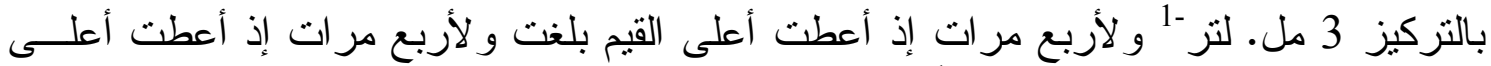

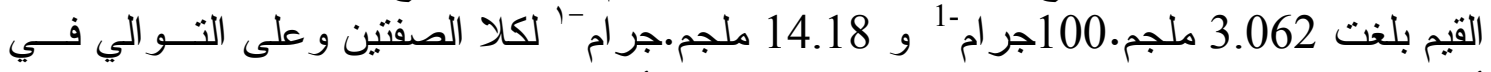

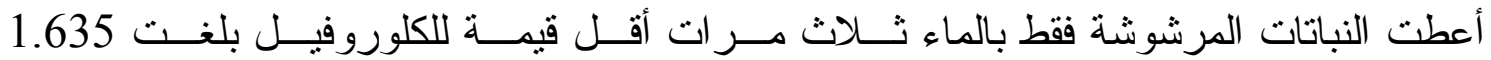

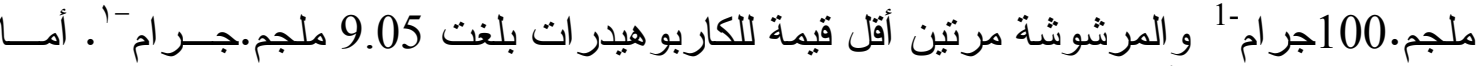

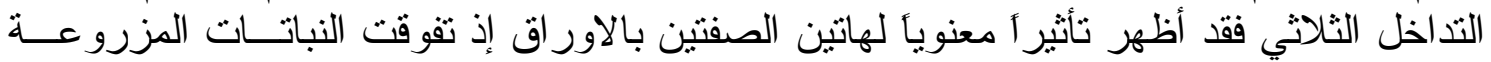

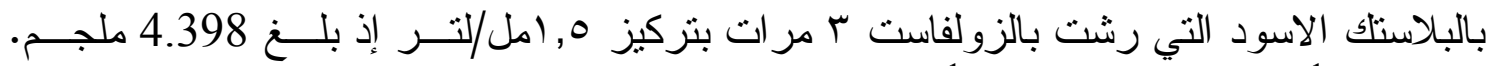

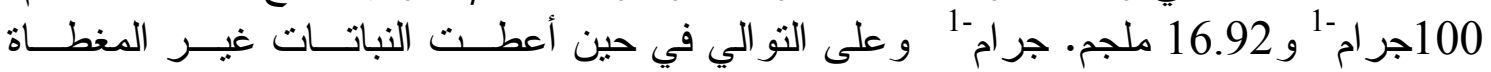

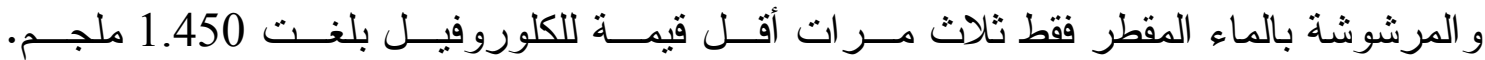
100جر ام -1 ولمرنين أقل قيمة للكاربو هيدر ات بلغت 6.45 ملجم. جر ام -1 . 
جدول 1. تأثير تغطية التربة وتركيزات وعدد مرات الرش بالكبريت السائل Zolfast وتداخلاتها في محتوى

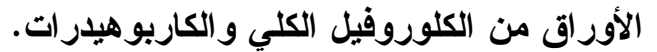

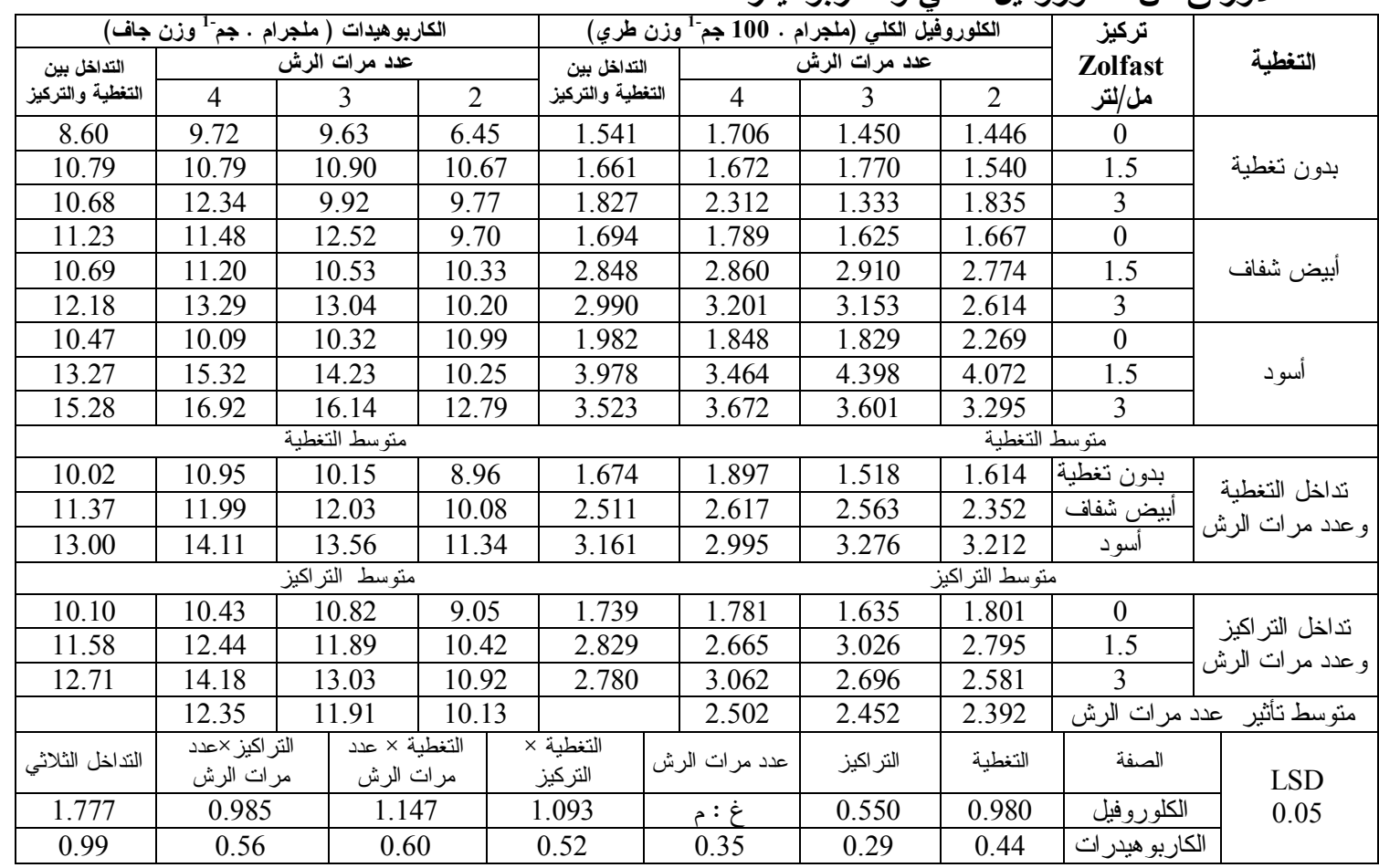

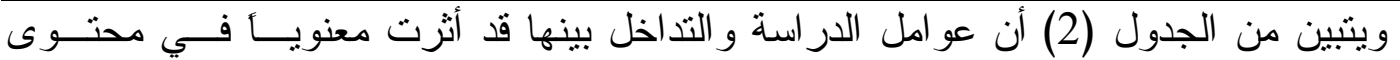

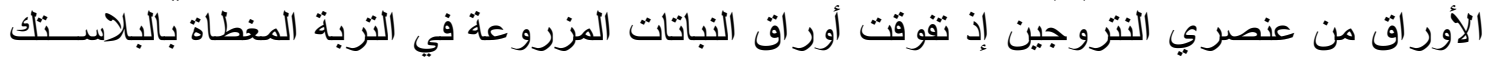

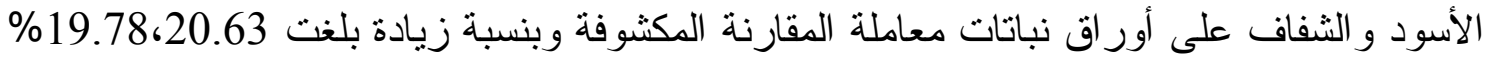

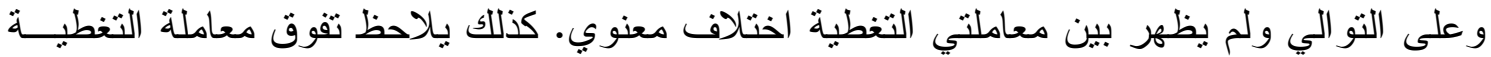

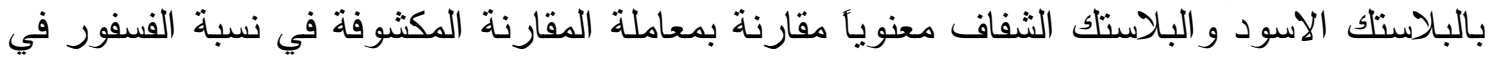

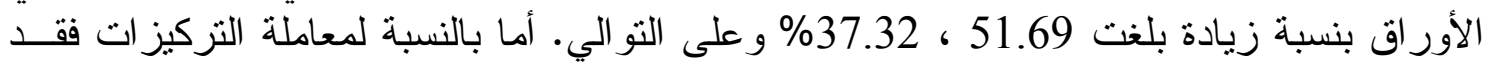

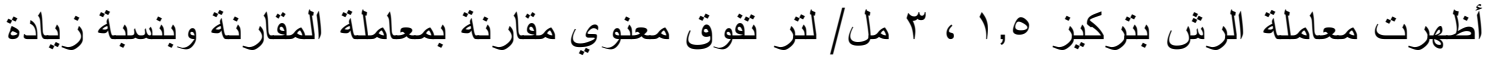

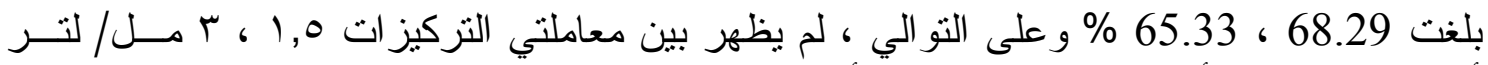

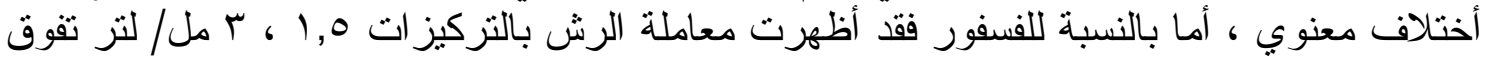

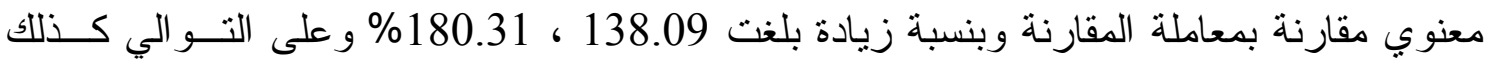

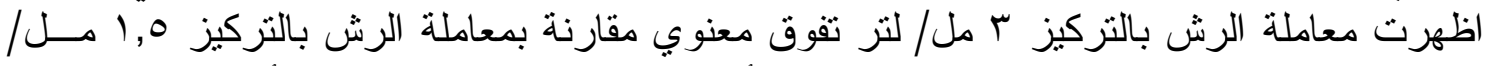

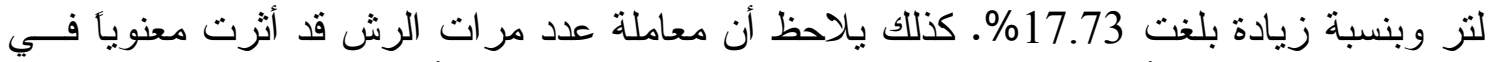

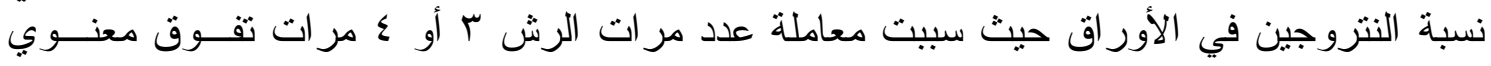

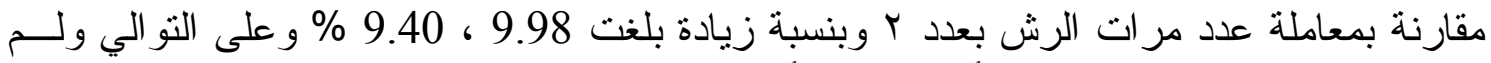

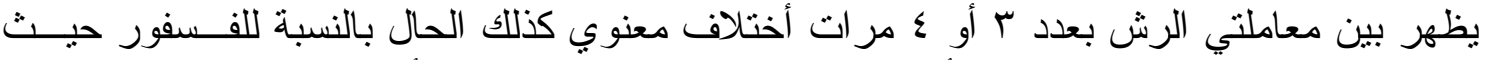

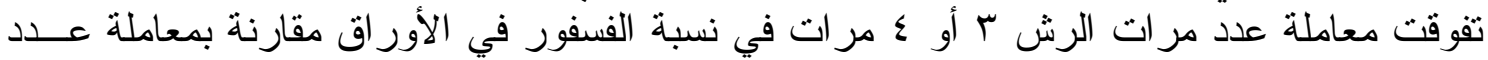

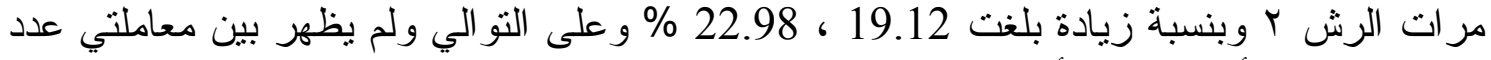

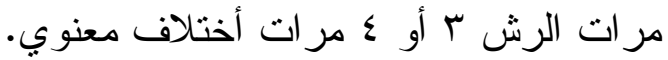

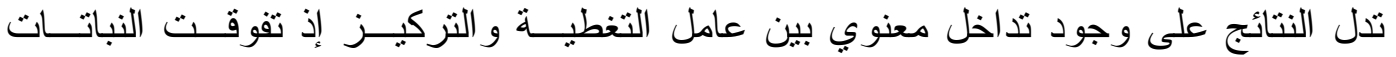

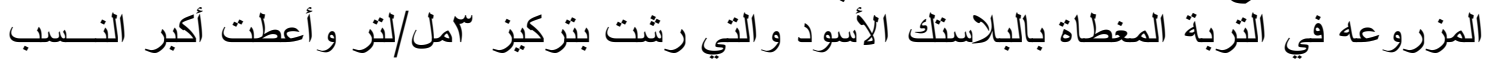
بلغت (3.63 ، 1.04)\% وعلى التو الي في حين أعطت النباتات المزروعه في التربة غير التئ المغطاة أقل النسب بلغت (1.74 ، 0.16) \% و و على التو الي. 


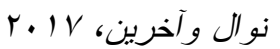

http://ajas.js.iknito.com/

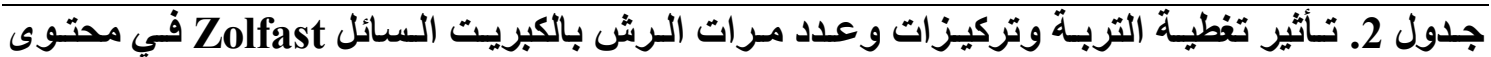

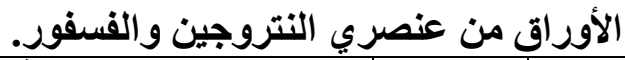

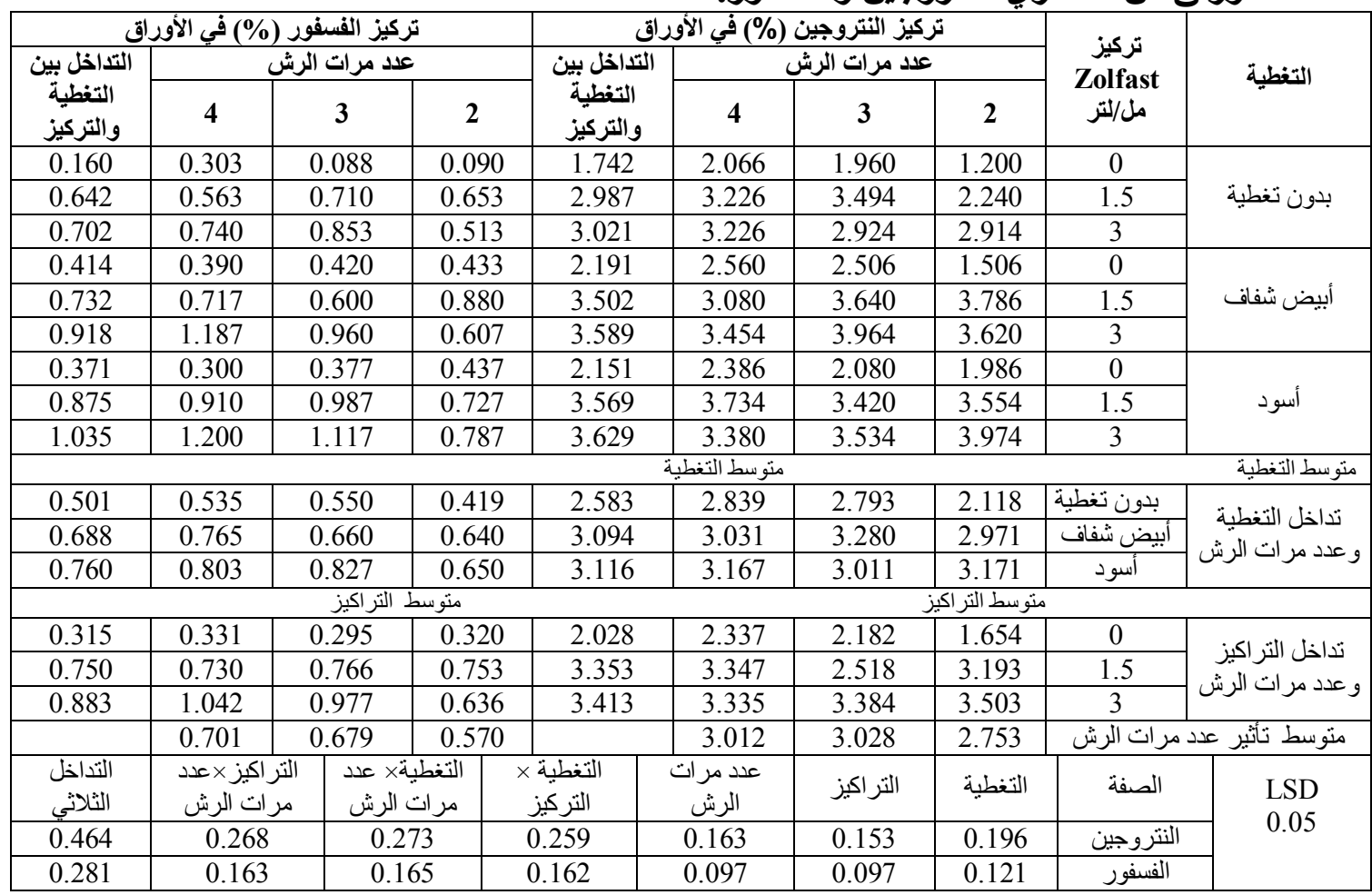

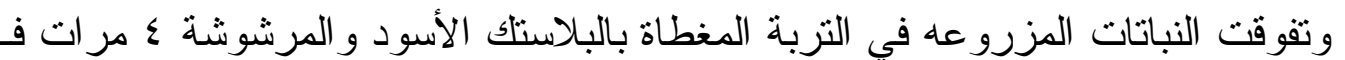

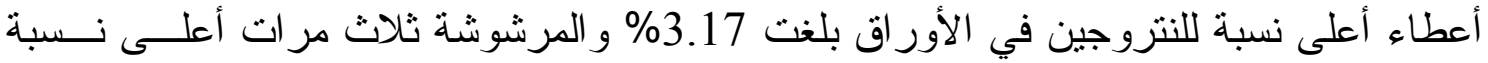
للفسفور بلغت 0.83\% في حين أعطت النباتات المزروعه في التزبة غير المغطاة و المرشوشة مرتين أقل النسب لهاتين الصفنتين بلغتا (2.12 ، 0.42)\% و على التو التي كما أظهر التداخل بـين

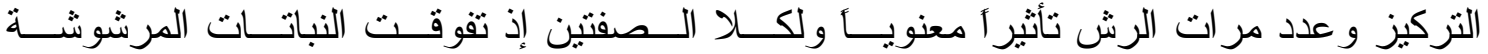

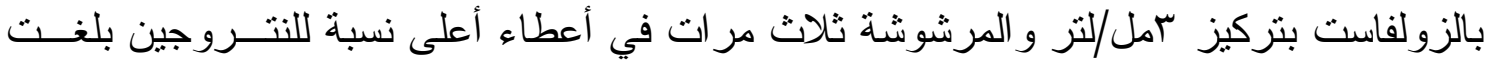

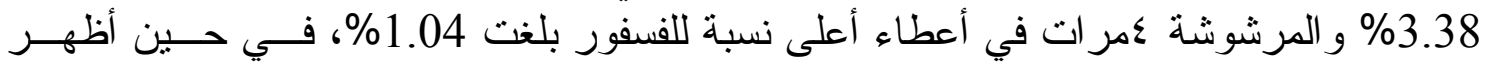
التداخل الثنلاثي بين المعاملات تفوق النباتات المزروعة في في التربة المغطاة بالبلاستك الأسود التي

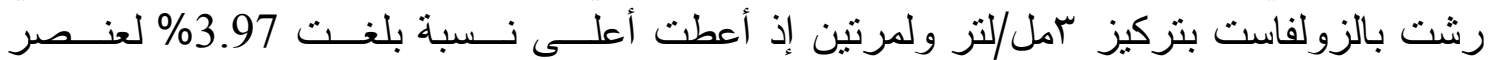

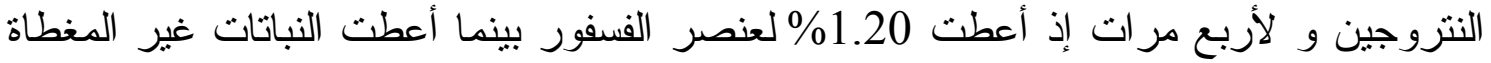
و المرشوشة بالماء المقطر مرتين أوطئ نسبة للنتروجين بلغت 1.20\% و المرشوشة ثلاث مر ات أوطئ نسبة للفسفور بلغت باء

) أن جميع عو امل الدراسة وتداخلاتها قد أثرت معنوياً في محتوى 3ويظهر من الجدول

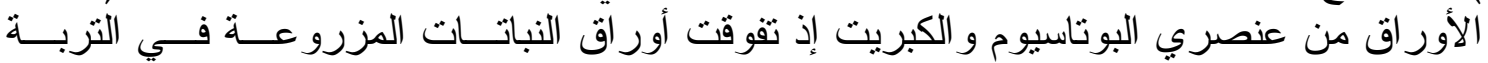

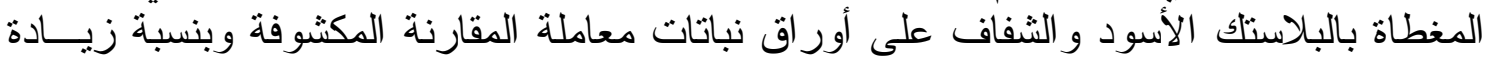
\% للغطاء الثفاف كذللك يظهر من الجـدول 11.76 \% بالنسبة للغطاء الأسود و 23.28بلغت

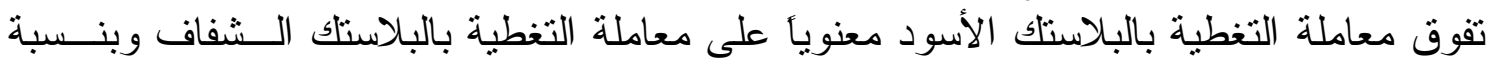

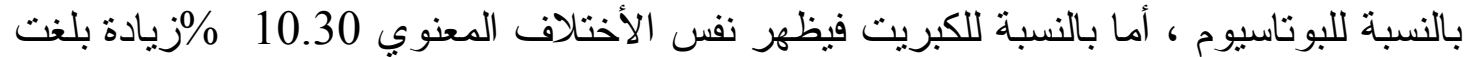

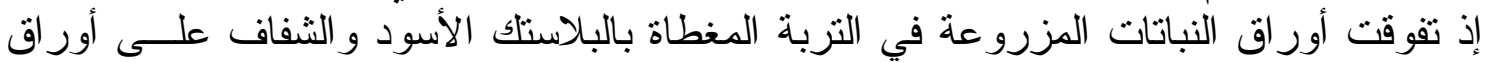

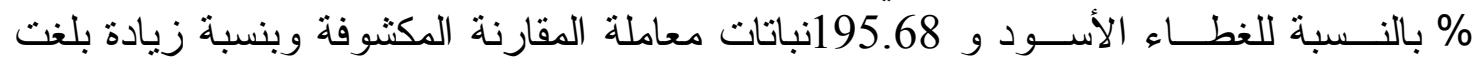


\% للغطاء الثفاف كذلك يظهر من الجدول تفوق معاملة التغطية بالبلاســانك الأســود 111.16

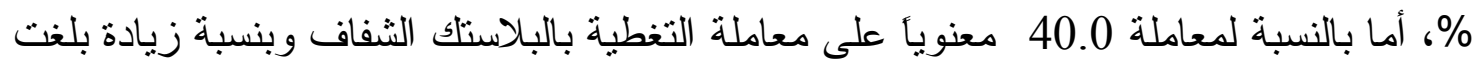
تركيز ات الرش فقد أظهرت معاملة التركيز ات

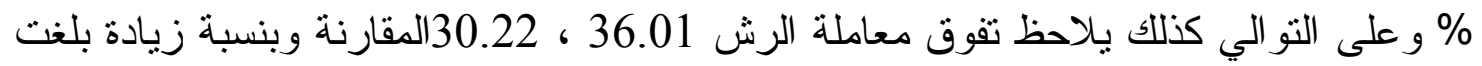

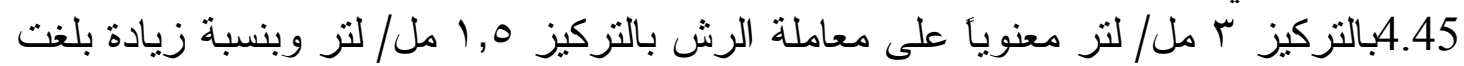

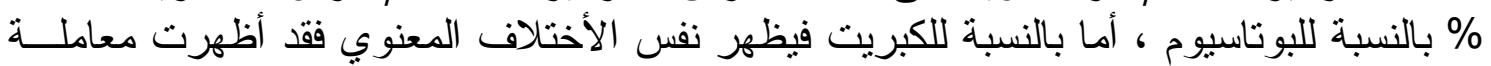

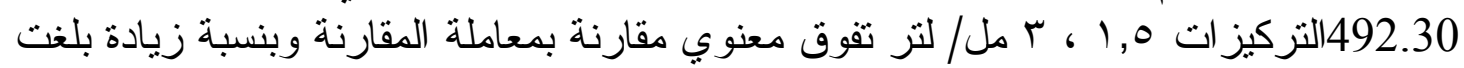

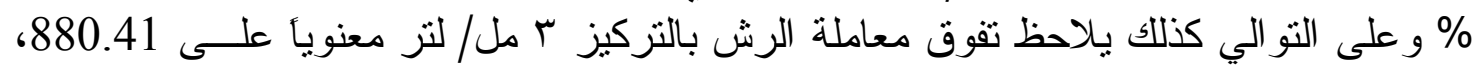

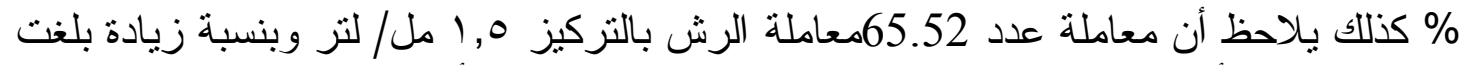

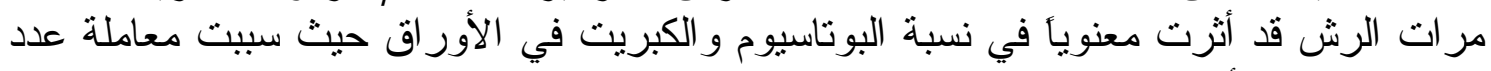

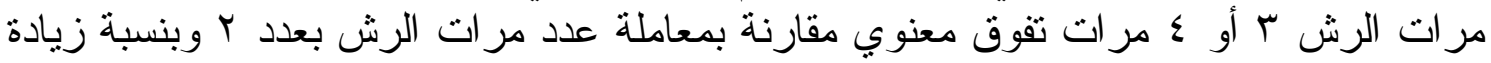

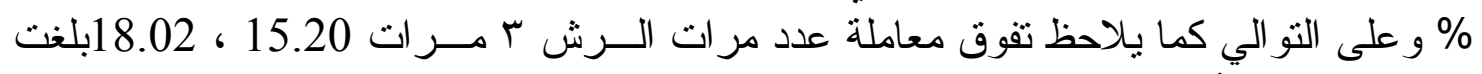

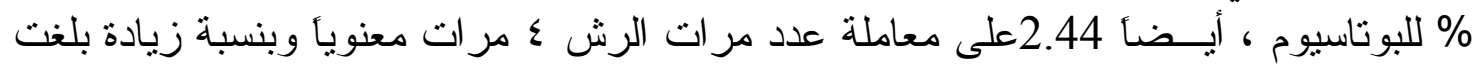

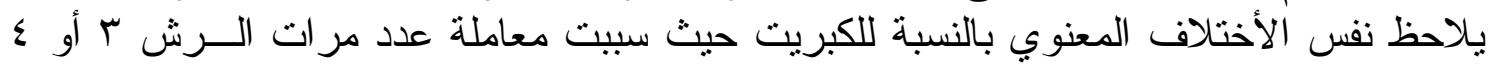

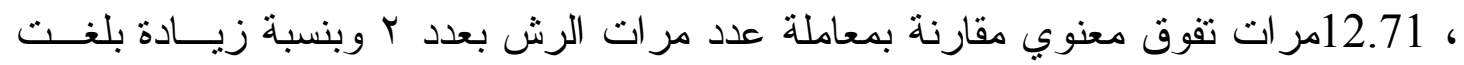

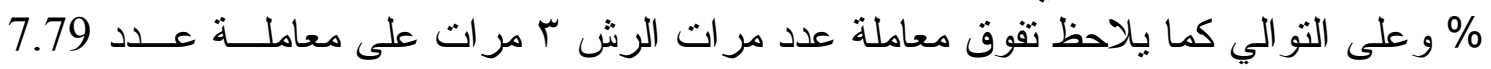

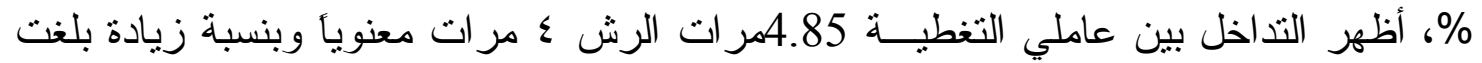

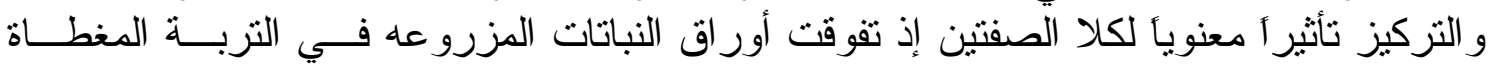

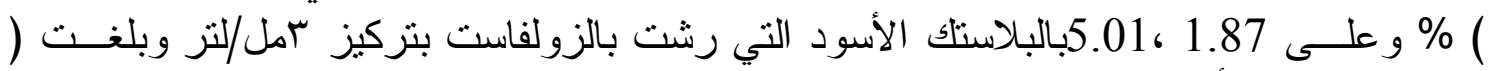

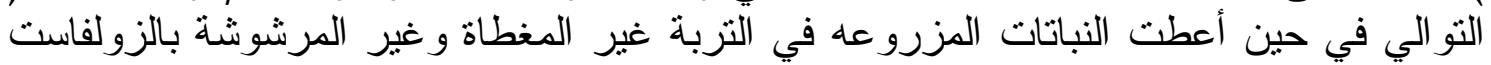

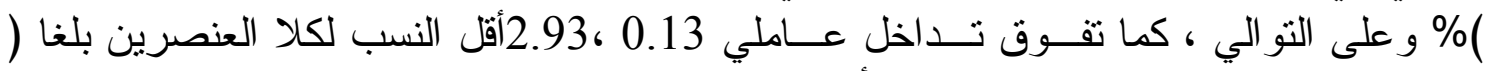

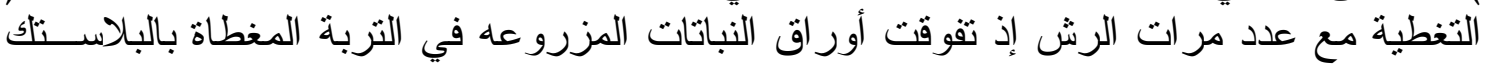

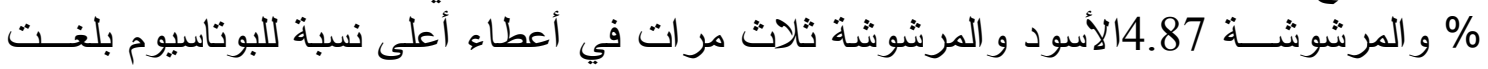

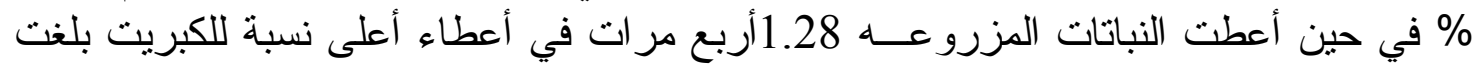
\% و المرشوشة ثاثل

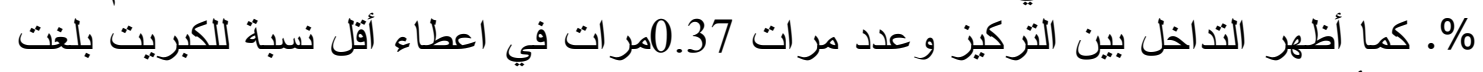

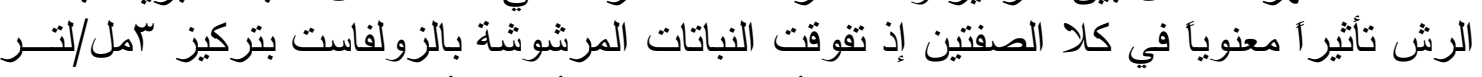

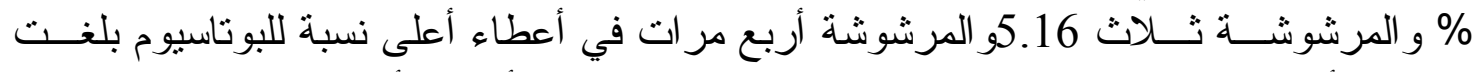

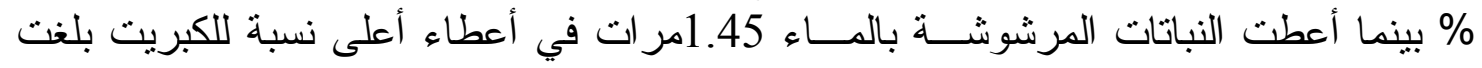

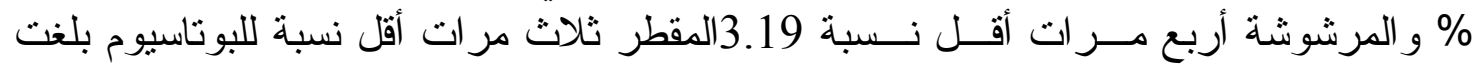

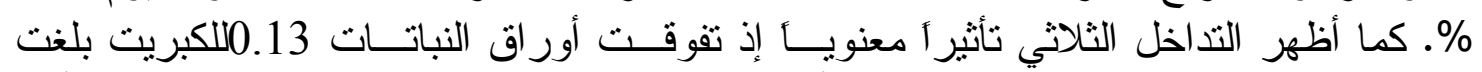

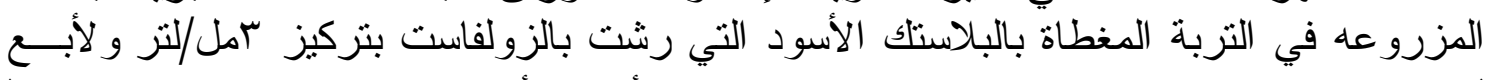

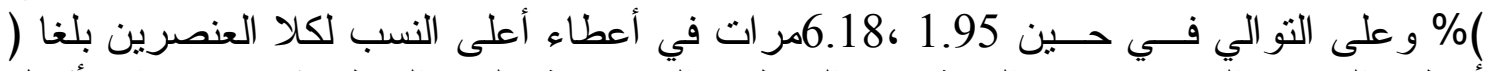

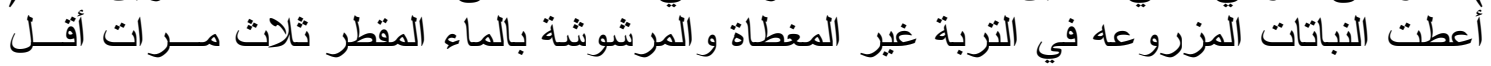

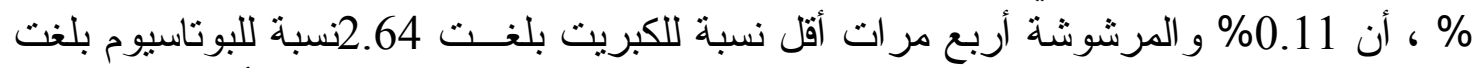

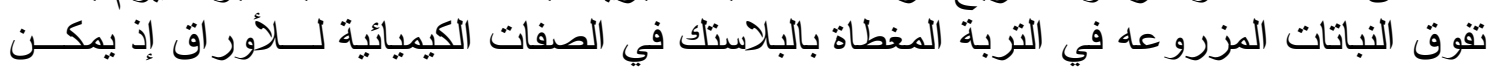

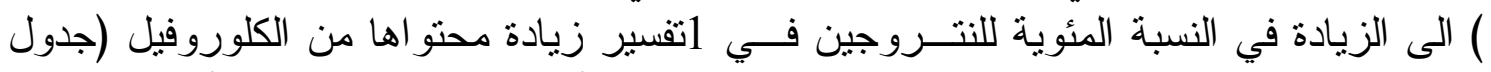

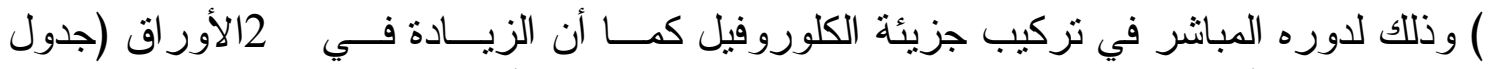

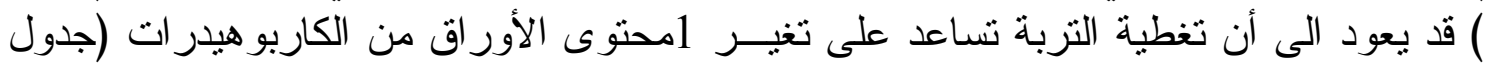




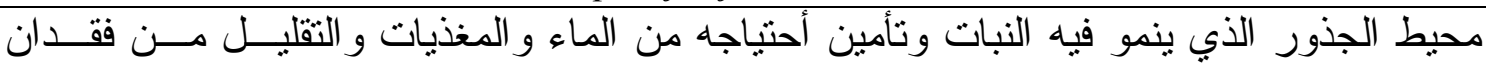

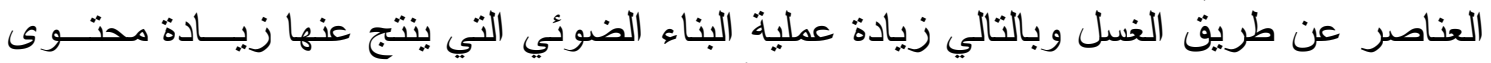

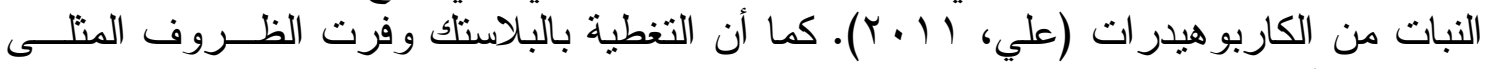

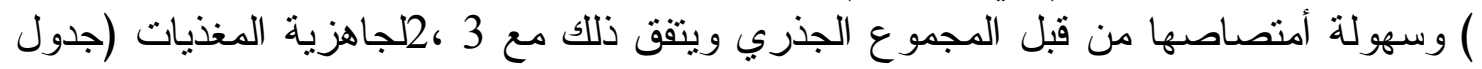

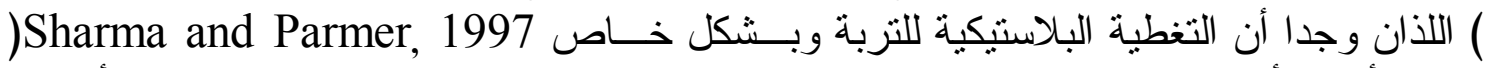

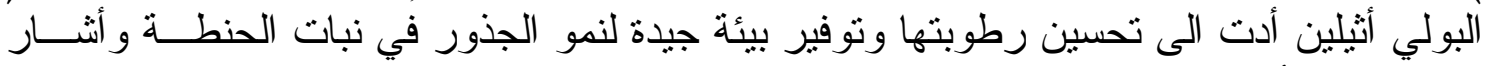

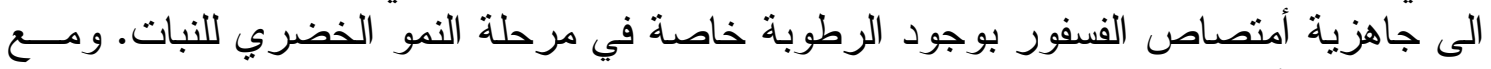

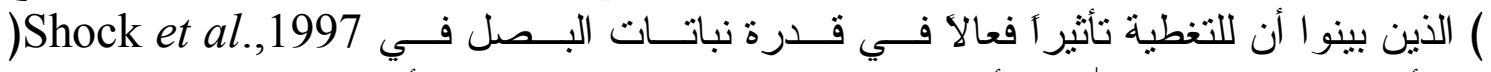

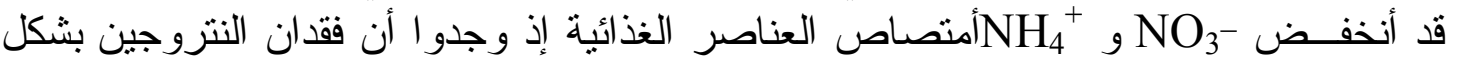

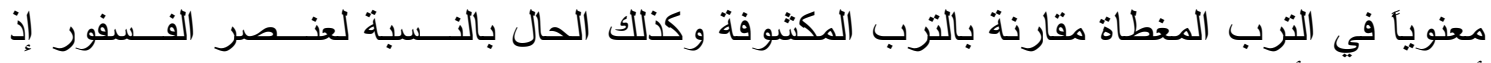

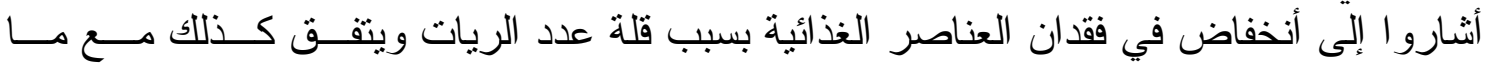
) في نبات الخيار .2008 و العبد الله ،

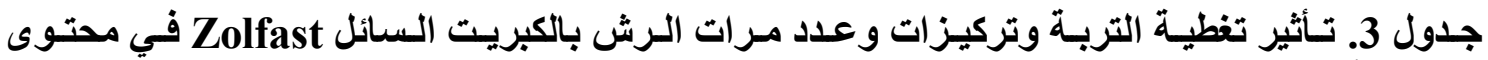

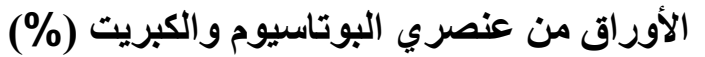

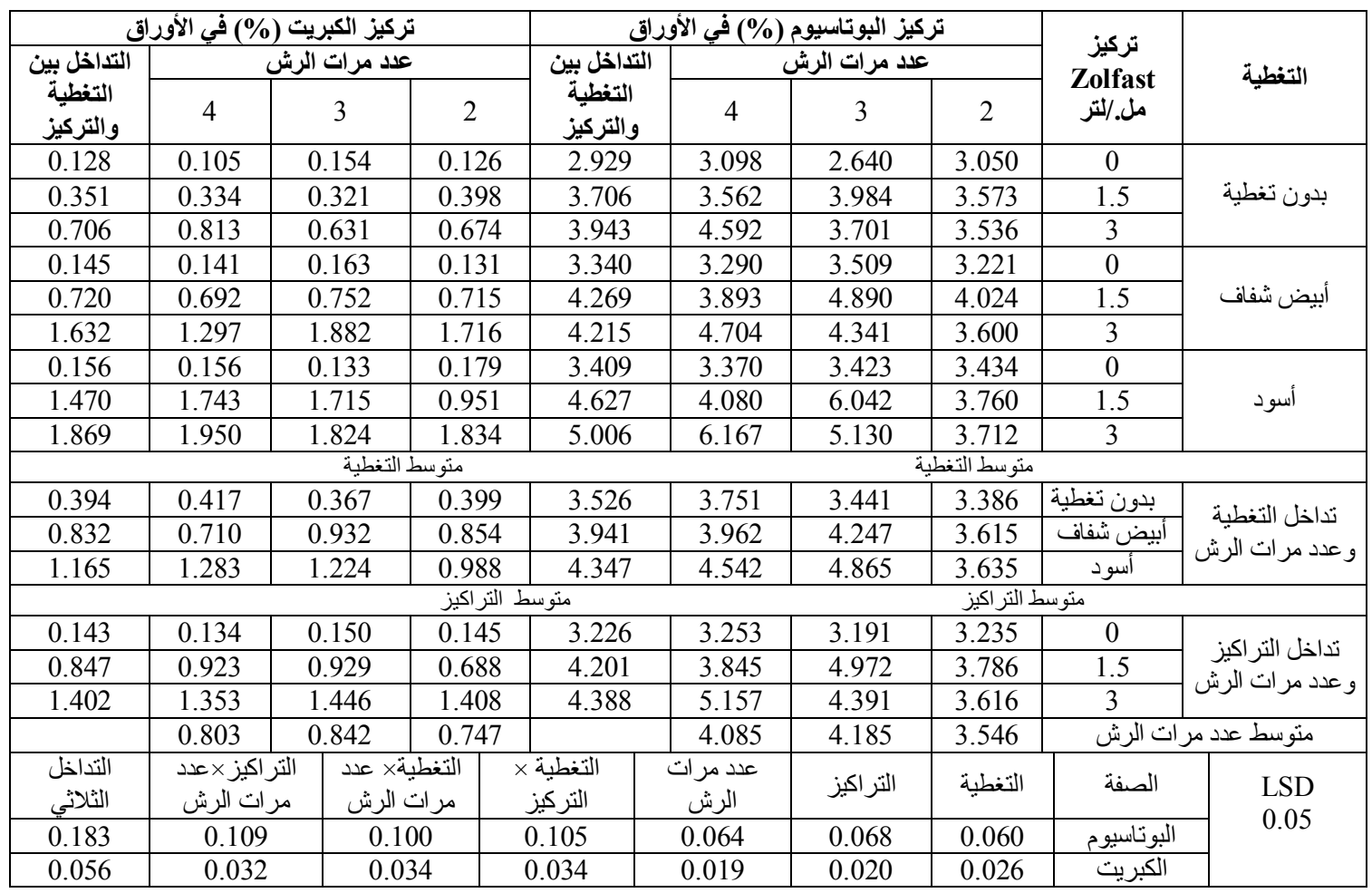

أما عن تزكيز وعدد مر ات الرش بالزولفاست في الصفات الكيميائيـــة فيـــدو أن تــأثير

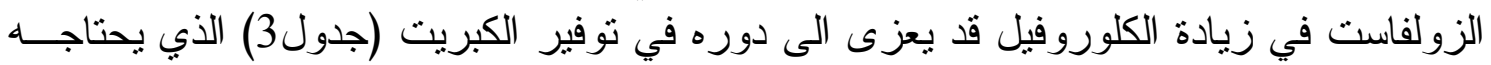

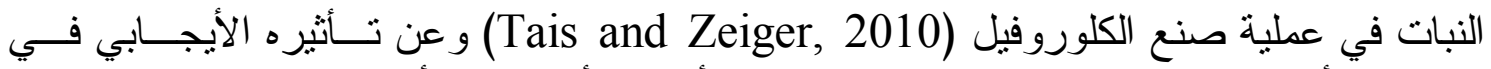

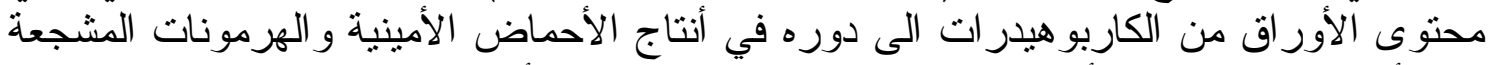

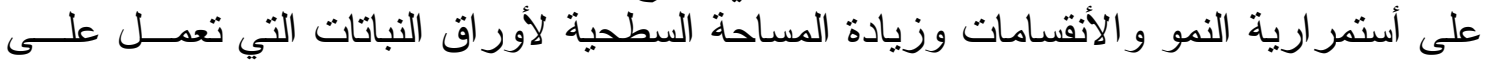

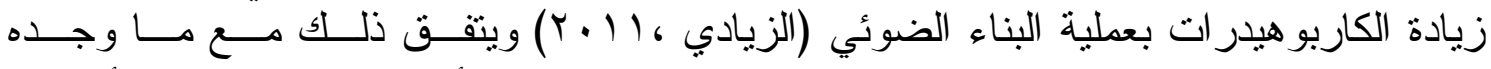
(Balkhi et. al., 2009) 


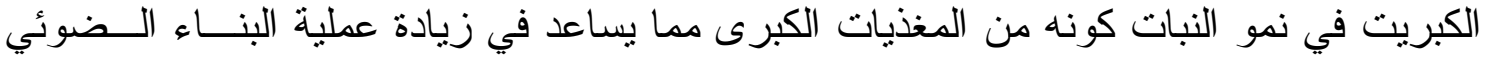

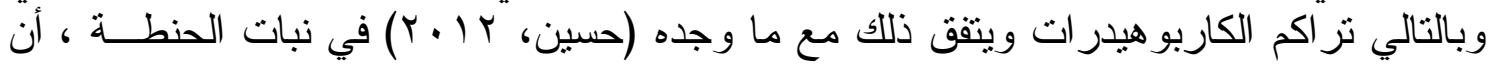

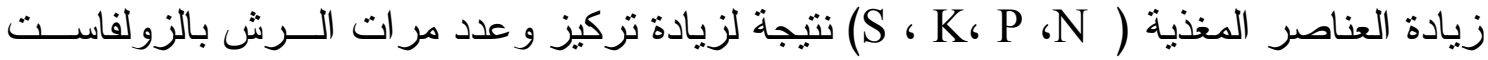

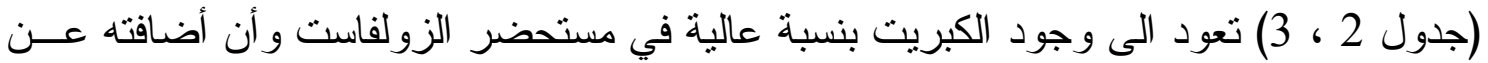

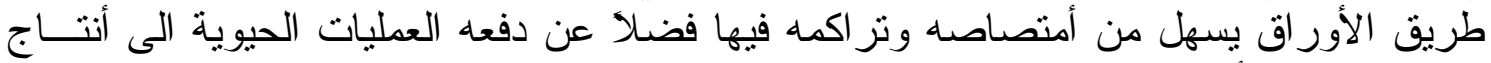

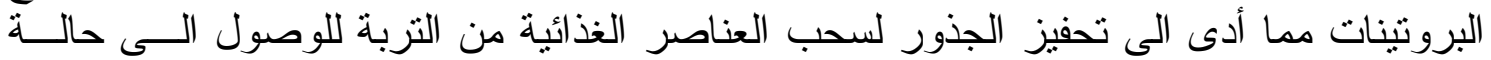

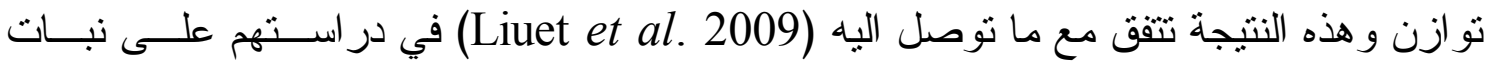

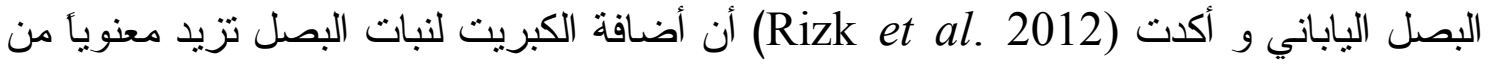

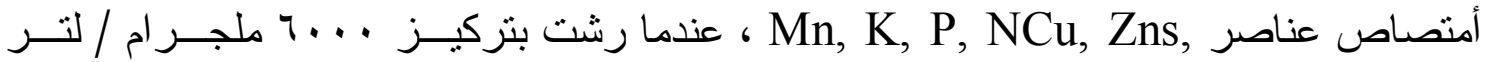

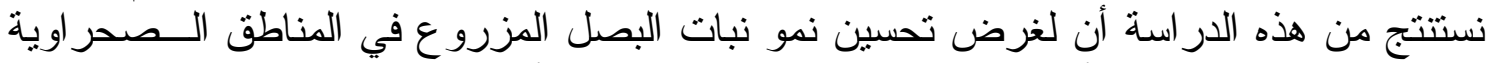

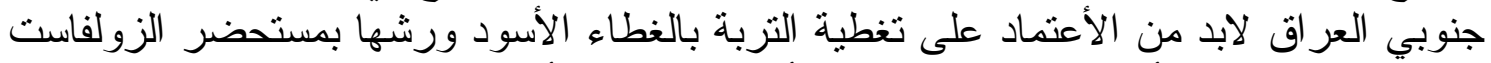

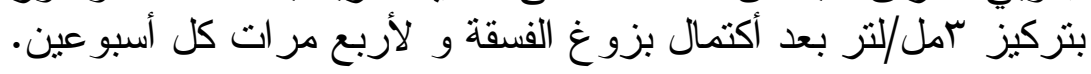

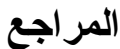

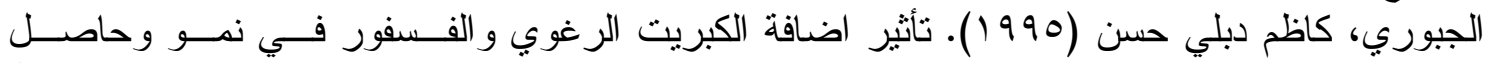

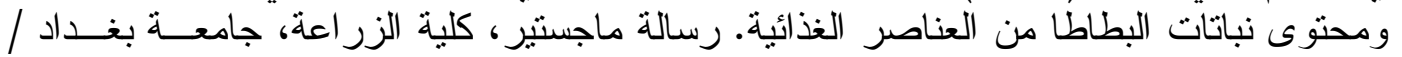

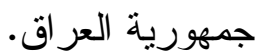

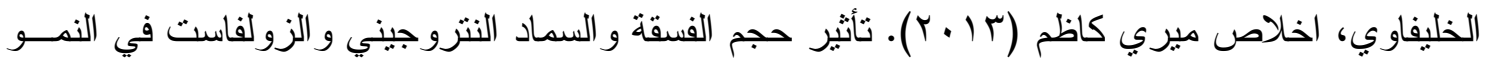

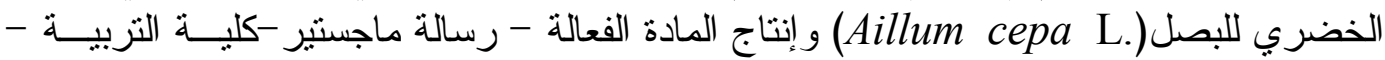
جامعة القادية /جمهورية العراق.

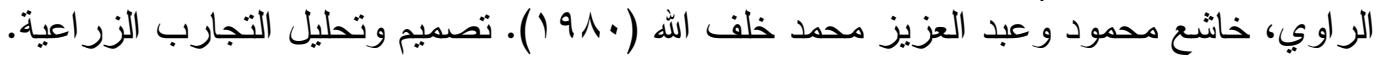

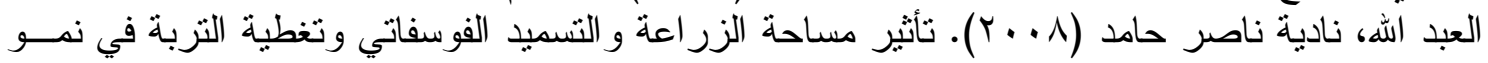

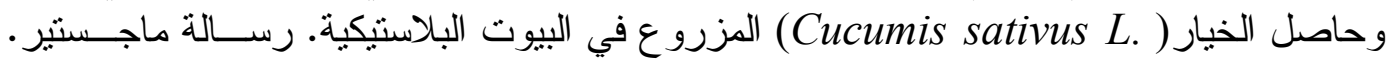

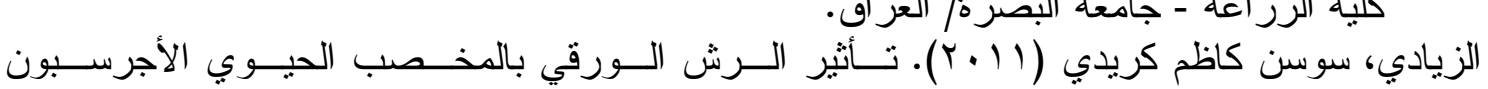

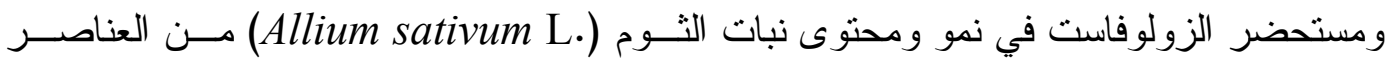

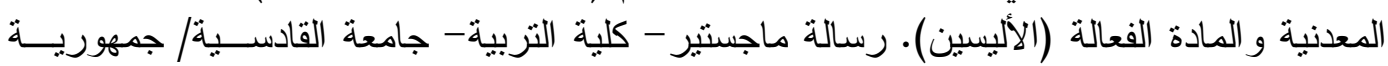

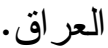
عباس، مؤيد فاضل و محسن جلاب عباس(1999). عناية وخزن الفاكهة و الخضر العملي. مطبعـة دار

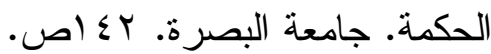

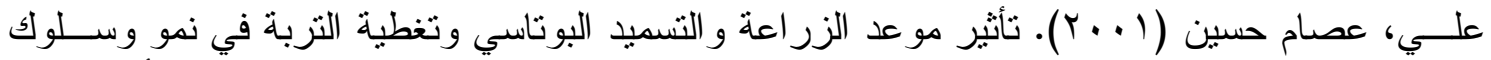

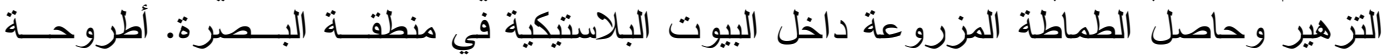

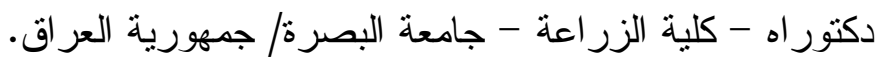

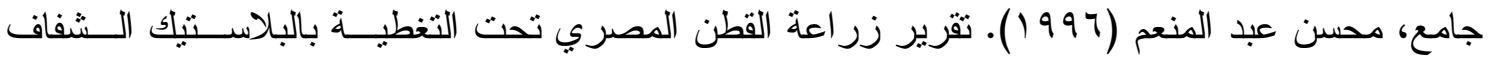

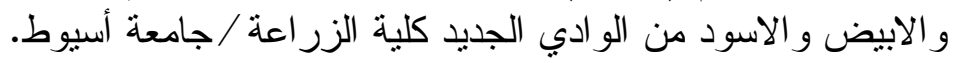

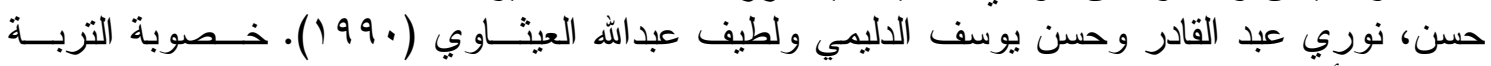

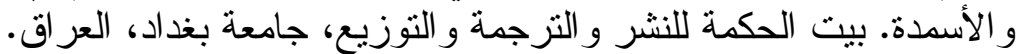

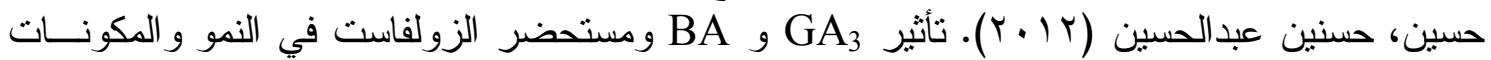

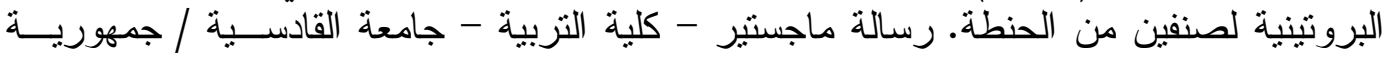

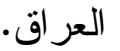

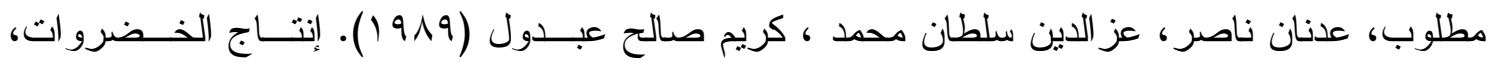

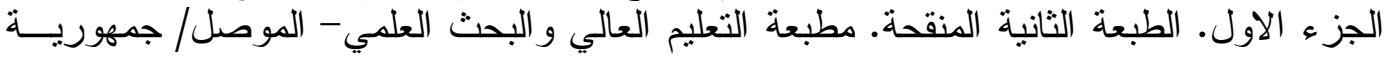

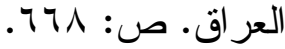


Al-Rawi, I.; Abd Al-Gafoor, A.; Dougramgi, J.; Hamdi, L. and Kayar S. (1984). Effect of Black plastic mulch on moisture and salt distribution through a soil profile. J. Agric. Water Resh. 19-42.

Al- Masoum, A. (1991). Effect of different mulch materials on soil temperature and yield of cauliflower. J. Emir. Agric. Sci., 3: 67-79

Balkhi, M.; Amin, S. and Aasood, A. (2009). Effect of aqueous sulfur dioxide on the biochemical and antioxidant properties of (Malva sylvestris.) Asian J. Environ. Sci., 3(2): 139-145.

Cannington, F.; Dugging, R.B. and Roan, R.C. (1975). Florida vegetable production using plastic film mulch with drip irrigation. Proc $12^{\text {th }}$ Natl, Agr, Plastics Congr. 11-15.

Cresser, M.S. and Parsons, J.W. (1979). Sulphuric-perchloric acid of digestion of plant material for determination of nitrogen, phosphorus, potassium, calicium and magnesium. Analytical Chimica Acta, 109: 431-436.

Dubois, M. K.; Crilles, K. A.; Hamiltor, J. K.; Rebers, D. A. and F. Smith (1956). Colorimetric method for determination of sugars and substance. Anal, Chem., 28 pp: 350-365.

Feucht, J. R. (2004). Mulches for home Grounds. CSU cooperative extension Horticulture. No.7.214.3.

Kaya, C.; Higgs, D. and Kinak, H. (2005). Influence of polyethylene mulch, irrigation regime and potassium rates on field cucumber yield and related trails. Journal of Plant Nutrition, 28 (10): 1739-1753.

Kirnak, H.; and Demirtas, M. N. (2006). Effects of different irrigation regimes and mulches on yield and macro nutrition levels of drip-irrigated cucumber under open field conditions. Journal of Plant.

Liu, S.; Feng, H. He; G. and Chen, Q. (2009). Effect of nitrogen and Sulfur interaction on growth and pungency of different pseudstem types of Chines spring onion (Allium fistulosum L.). Scienta Horticulture, 121(1):12 - 18.

Murphy, T.; and Riley, J. R. (1962). A modified single solution method for the determination of phosphate in natural waters. Anal. Chem. Acta, 27,: 31-36.

Page, A. L.; R. H. Miller, R. H.; and Keeney, D. R. (1982). Methods of Soil Analysis Part 2, $2^{\text {nd }}$ Ed. Madison Son, Wisconson, USA: P. 1159.

Qotob, A. M.; S. A. Mohammed; A. 1. Amin ; G Shaker and A. A El-Masry (2016). Evaluation of elemental sulphur and different nitrogen fertilizers on biochemical component of (Allium cepa L.) plant. Advances in Environmental Biology, 10 (11) : 10-17.

Rizk, F.A; A.M. Shaheen, E.H. Abd El- Samed and O.M. Sawan (2012). Effect of different nitrogen plus phosphorus and sulphur fertilizer levels on growth, yield and quality of onion (Allium cepa L.) J. Appl. Sci. Res., 8 (7): $3353-3361$.

Roberts, B.; Warren, and Jeffery, A. Anderson (1994). Canopy shad and soil Mulch affect yield and solar injury of Bell Pepper. Hort Sci. V. 39 (4): 258261.

Sharma, P. K. and Parmer, D. K. (1997). Effect of phosphorus and mulching on the uptake of secondary Nutrients and productivity of wheat atdifferent 
Growth stages on an Alfisol from western Himalayas. Journal of the Indian society of soil science, V.46 (1),: 67-70.

Sherestha, H. (2007). A plant monograph on onion (Allium cepa.). The school of pharmaceutical and biomedical sciences. Roll No. 11/2004 Pokhara Univ. Nepal.

Shock, C. C; Hodson, J. H.; Seddigh, M.; Shock, B. M.; Stieber, T. D. and Saunders, L. D. (1997). Mechanical straw Mulching of Irrigatio Furrows: Soil Erosion and Nutrient: losses. Published in Agron. J. 89: 887-893.

Spiess, E.; (1994). Mulch treatment, in potato growing, Experiment Switzerland. Kartoffelbau, 45 (3): 48-52.

Streck, N.A.; Schneider, F.M. and Buriol, G.A. (1996). Soil heating by solarization inside plastic greenhouse in Santa Maria, Rio do sul, Brazil. Agricultural and forest Meteorology, 82. 73-82.

Taiz, L. and Zeiger, E. (2010). Plant Physiology. $5^{\text {th }}$ ed. Sinauer Associoates, Publishers. Sunderland, Massachusetts.

Watt, B. K.; and Merril, A.L. (1963). Composition of food. U.S. Dept. Agr. Hand book N. 8. 190 P. 


\title{
Effect of Mulching, Concentration and the Number of Sprays with Liquid Sulfur ZOLFAST in Chemical Contents of Leaves of the Local Red Onion Cultivar
}

\author{
Nawal M. Hmood ; A. A. Abdulla and A. J. Fahad \\ Department of Horticultural Science and Land Scape - College of Agriculture - Basrah \\ University- Iraq.
}

\begin{abstract}
A field experiment was conducted during the agricultural season 20162015 in the project of cultivating onion using the modern technologies of the Directorate of Basrah Agriculture in Khor al-Zubair - Basrah in order to study the effect of the mulching, concentration and the number of sprays with liquid sulfur zolfast in the growth and yield of the local red onion cultivar.

The experiment included three factors: the Interactions between three types of mulch (white, black as well as without mulch) and spraying with liquid sulfur Zolfast with three concentrations $(0,1.5$ and $3 \mathrm{ml} \backslash 1)$ twice or three or four times.

The experiment was implemented as Factorial Experiment Split Split Plot Design by Randomized Complete Block Design (R.C.B.D.) in three replicates and the arithmetic means of the treatments were compared using the least significant difference test (L.S.D at 0.05).

The results showed that there is a significant increase in total chlorophyll, carbohydrate, nitrogen, potassium $\%$ and sulpher contents in the plants leaves mulched with black plastic as compared to non-mulched plants. Mulching with black or transparent white plastic show significant increase in phosphorus contents as well.
\end{abstract}

Plants sprayed with zolfast at concentrations of $3 \mathrm{ml} \backslash 1$ and $1.5 \mathrm{ml} \backslash 1$ were superior in total chlorophyll content and percentage of nitrogen while those sprayed with concentration of $3 \mathrm{ml} \backslash 1$ contained high content of carbohydrates, phosphorus, potassium and sulfur.

The leaves of plants that were sprayed four times with zolfast significantly exceeded in their carbohydrate and potassium content, while those sprayed three times excelled in percentage of sulfur as such those were sprayed three or four times excelled in percentage of nitrogen \& phosphorus.

The interactions between the studied factors showed a significant effect on most of the traits. 